\title{
ON A CLASS OF CONFORMAL METRICS
}

\author{
MAURICE HEINS \\ Dedicated to my Japanese colleagues on the occasion of the Symposium \\ on the Theory of Functions held by the Theory of Functions Branch of \\ the Mathematical Society of Japan at Nagoya, July 3-5, 1961.
}

Last year when I was preparing for course lectures the work of Ahlfors [1] which establishes that the Bloch constant is at least as large as $\sqrt{3} / 4$, it appeared to me that the resources of the theory of metrics of negative curvature offered rich possibilities from a function-theoretic point of view. The parallelism between certain properties of subharmonic functions and those of the metrics introduced by Ahlfors [1] is so striking that we are led to ask whether one can introduce a class of metrics including the metrics of Ahlfors for which not only does a Schwarz-Pick-Ahlfors lemma hold, but also requirements of differentiability disappear, as in the modern theory of subharmonic functions. We shall define such a class. To it part of the apparatus of the theory of subharmonic functions, including the use of Perron families, may be transplanted. Among the results that we obtain is the conclusion that the inequality of the Schwarz-Pick-Ahlfors lemma is strong throughout for an admitted metric distinct from the hyperbolic metric [\$7]. This theorem will permit us to show that the Bloch constant is actually greater than $\sqrt{3} / 4[\$ 36]$.

A metric of special interest from the point of view of the theory of conformal maps of Riemann surfaces is one having constant curvature -4 save for a discrete set at each point of which it vanishes. Metrics induced by a conformal map from a hyperbolic metric are of this type. We shall see that the distribution of the zeros of the derivative of a non-constant bounded analytic function with domain $\Delta=\{|z|<1\}$ may be characterized in terms of such a metric [\$29]. In this connection, the following result deserves mention: The distribution of points in $\Delta$ at which a Lindelöfian map with domain $\Delta$ is ramified, multiplicities being taken into account, is no more general than the distribution of the ramification points of a non-constant bounded analytic function with domain $\Delta[\S 30]$.

Received July 4, 1961. 
It does not appear to be desirable to summarize other results of the present study without the benefit of a preliminary statement of the terms and concepts we shall employ. For this reason, we turn directly to our study.

\section{Chapter 1. $S-K$ metric}

1. Our first step will be to pass from the formulation of Ahlfors to a more general one that looks to the theory of subharmonic functions for its model. Let $F$ denote a Riemann surface, and let $S$ denote the basic family of homeomorphisms $\sigma$ of plane regions into $F$ which defines the conformal structure of $F$. We adopt a general point of view and define a conformal metric $\lambda$ on $F$ to be a non-negative density of degree one. We do not impose any continuity restrictions. To be precise, we mean that $\lambda$ is a map with domain $S$ which has the following two properties:

(a) $\lambda_{\sigma}$, the image of $\sigma \in S$ with respect to $\lambda$, is a function having the same domain as $\sigma$ and taking finite non-negative values.

(b) Let $\sigma, \tau \in S$ and let their images have intersection $O \neq \varnothing$. Let $\theta$ denote the univalent analytic function connecting $\sigma$ and $\tau$, defined by

$$
\theta=\left\{\left(a^{-1}(p), \tau^{-1}(p)\right) \mid p \in O\right\} .
$$

Then

$$
\lambda_{s}(z)=\lambda_{-}[\theta(z)]\left|\theta^{\prime}(z)\right|
$$

for each $z$ in the domain of $\theta$.

Given a uniformizer $\varphi$ of $F$ ( = univalent conformal map of a plane region into $F$ ), there exists a unique extension of $\lambda$ to $S \cup\{\varphi\}$ which is a conformal metric relative to $S \cup\{\varphi\}$. We denote the image of $\varphi$ with respect to this extension by $\lambda_{\varphi}$ and term it the $\varphi$-scale of $\lambda$.

If for some uniformizer $\varphi$ satisfying $\varphi(a)=p, \lambda_{p}(a)=0$, then for every uniformizer $\psi$ whose image contains $p, \lambda_{\psi}(b)=0, \psi(b)=p$. In this case, we say that $\lambda$ vanishes at $p$.

If $\mu$ is also a conformal metric on $F$ and $\lambda_{p}(a) \leq \mu_{\varphi}(a)$, then $\lambda_{\psi}(b) \leq \mu_{\psi}(b)$. In this case, we say that $\lambda$ does not exceed $\mu$ at $p$. To say " $\lambda$ does not exceed $\mu$ on $F$ " denoted " $\lambda \leq \mu$ " means that $\lambda$ does not exceed $\mu$ at each point of $F$. The strong inequalities at a point and on $F$ are similarly defined.

By $\max \{\lambda, \mu\}$ is meant the map with domain $S$ which assigns to $\sigma$ the 
function $\max \left\{\lambda_{\sigma}, \mu_{s}\right\} . \quad \max \{\lambda, \mu\}$ is a conformal metric, and if $\varphi$ is a uniformizer, $\max \{\lambda, \mu\}_{p}=\max \left\{\lambda_{p}, \mu_{p}\right\}$.

If $P$ is a non-negative finite-valued function on $F$, then $P \lambda$ will denote the function with domain $S$ which assigns to $\sigma$ the function $(P \circ \sigma) \lambda_{s}$. It is a conformal metric.

If $\lambda_{p}$ is upper semi-continuous at $a$, then $\lambda_{\psi}$ is upper semi-continuous at $b$. In this case, we say that $\lambda$ is upper semi-continuous at $p$. If $\lambda$ is upper semicontinuous at each point of $F$, we say that $\lambda$ is upper semi-continuous on $F$. Similarly, if $\lambda_{\text {p }}$ is of class $C^{(k)}$ (or real analytic) in some neighborhood of $a$, the same holds for $\lambda_{\psi}$ in some neighborhood of $b$, and correspondingly, we say that $\lambda$ is of class $C^{(k)}$ (resp. real analytic) in a neighborhood of $p$. It is obvious what is meant by saying that $\lambda$ is of class $C^{(k)}$ (resp. real analytic) on $F$.

If $\lambda$ enjoys adequate smoothness properties relative to a point $p \in F$, then we may introduce the Gaussian curvature of $\lambda$ at $p$ as follows. Suppose that $\lambda$ is of class $C^{\prime \prime}$ in some neighborhood of $p$ and that $\lambda$ does not vanish at $p$. Then for every uniformizer $\varphi$ for which $p$ lies in the image of $\varphi$ and $\varphi(a)=p$, the value of

$$
\frac{-\Delta \log \lambda_{p}(a)}{\left[\lambda_{\xi}(a)\right]^{2}}
$$

is independent of $\varphi$ and is termed the Gaussian curvature of $\lambda$ at $p$. It will be denoted by $K(p ; \lambda)$. Metrics $\lambda$ that are $C^{\prime \prime}$ and such that $K(p ; \lambda)=-4$, $p \in F$, are of particular interest from a function-theoretic point of view. The hyperbolic metric $\lambda$ on $\{|z|<1\}$ given by $\lambda_{l}(z)=\left(1-|z|^{2}\right)^{-1}$ is of special importance. It is a metric of constant curvature -4 .

We are now in a position to define an $S-K$ metric. (" $S-K$ " is intended to convey "curvature subordinate to -4 ". The significance of this nomenclature will become clear after Theorem 2.1.) We should indicate that we are concerned with metrics that reduce in the $C^{\prime \prime}$ case to metrics with curvature no greater than -4 at points that are not zeros of the metrics. We say that a metric $\lambda$ is an $S-K$ metric provided that the following two conditions are fulfilled :

(a) $\lambda$ is upper semi-continuous on $F$.

(b) Let $\varphi$ denote a uniformizer with domain $\{|z|<1\}$, let $u=\log \lambda_{\text {s, }}$, and let $m(r ; u)$ denote the circumferential mean 


$$
(2 \pi)^{-1} \int_{0}^{2 \pi} u\left(r e^{i \theta}\right) d \theta, \quad 0<r<1
$$

Then, when $\lambda_{p}(0)>0$,

$$
\liminf _{r \rightarrow 0} r^{-2}[m(r ; u)-u(0)] \geq\left[\lambda_{p}(0)\right]^{2} .
$$

It is to be observed that the left-hand side of (1.4) is just one fourth of the lower generalized Laplacian of $u$ at 0 . If $\lambda_{p}$ is of class $C^{\prime \prime}$ in a neighborhood of 0 , the condition (1.4) is equivalent to

$$
\Delta u(0) \geq 4\left[\lambda_{p}(0)\right]^{2} .
$$

2. We now turn to the study of properties of $S-K$ metrics. This part of our investigation is strongly motivated by the analogy between $S$ - $K$ metrics and subharmonic functions. We seek to adapt methods of the theory of subharmonic functions to our class of metrics. A first task confronting us is the extension of Ahlfors's maximum principle to $S-K$ metrics.

Suppose that $\Omega$ is a relatively compact region of $F$ and that $\lambda$ is an $S-K$ metric on $\Omega(\Omega$ itself being considered as a Riemann surface). Let $\mu$ denote a $C^{\prime \prime}$ conformal metric on $\Omega$ which vanishes nowhere and has constant Gaussian curvature taking the value -4 . The following is a very evident generalization of the maximum principle used by Ahlfors [1, p. 360].

THEOREM 2.1: If

$$
\limsup _{p} \lambda / \mu \leq 1, \quad p \in \operatorname{fr} \Omega,
$$

then

$$
\lambda \leq \mu .
$$

Here $\lambda / \mu$ is the scalar function on $\Omega$ defined by

$$
\frac{\lambda}{\mu}=\left\{\frac{\lambda_{\rho} \circ \varphi^{-1}}{\mu_{\rho} \circ \varphi^{-1}} \mid \varphi \text { a uniformizer for } \Omega\right\} \text {. }
$$

The possibility that fr $\Omega$ may be empty is allowed. Thanks to the boundary condition (2.1) and the upper semi-continuity of $\lambda / \mu$, we see that if (2.2) did not hold, then $\lambda / \mu$ would possess a maximum greater than one at a point $q \in \Omega$. Let $\varphi$ denote a uniformizer with domain $\{|z|<1\}, \varphi(0)=q$, and let $w=\log \lambda_{\text {, }}$ $-\log \mu_{\varphi}$. Now $w$ has a positive absolute maximum at 0 . Hence 


$$
\limsup _{r \rightarrow 0} r^{-2}[m(r ; w)-w(0)] \leq 0 .
$$

On the other hand, from (1.4) and the hypothesis on $\mu$ we have

$$
\liminf _{r \rightarrow 0} r^{-2}[m(r ; w)-w(0)] \geq\left[\lambda_{\vartheta}(0)\right]^{2}-\left[\mu_{\vartheta}(0)\right]^{2}>0 .
$$

The contradiction is immediate.

3. We pursue the suggestive analogy between the theory of subharmonic functions and $S$ - $K$ metrics and first ask whether the property stated in Theorem 2.1 serves as a basis for an equivalent definition of an $S$ - $K$ metric.

Given an arbitrary conformal metric $\lambda$ on a Riemann surface $F$ and a nonempty set $E \subset F$, it will be convenient to introduce $\lambda^{E}$, the restriction of $\lambda$ to $E$, defined as follows. Given $\sigma \in S$, let $\widetilde{\sigma}$ denote the restriction of $\sigma$ to $\sigma^{-1}(E)$ and let $\lambda^{E}$ denote the map whose domain is the set of the non-empty $\widetilde{\sigma}$ which maps $\widetilde{\sigma}$ onto the restriction of $\lambda_{\sigma}$ to the domain of $\widetilde{\sigma}$. If $E$ is a region, then $\lambda^{k}$ is a conformal metric on $E$ considered as a Riemann surface. For a general $E$, by a conformal metric on $E$ is meant the restriction to $E$ of some conformal metric on $F$. The meanings of continuity and non-vanishing for such a metric are obvious.

Let us now suppose that $\lambda$ is a conformal metric on $F$ and that $\lambda$ satisfies the following two conditions: (a) $\lambda$ is upper semi-continuous on $F$. (b) For each $p \in F$, there exists a relatively compact neighborhood $V$ of $p$ such that, whenever $\mu$ is a $C^{\prime \prime}$ conformal metric with constant Gaussian curvature - 4 on a region $\Omega, p \in \Omega \subset V$, and $\mu$ satisfies

$$
\lim \sup _{q} \lambda^{2} / \mu \leq 1, \quad q \in \operatorname{fr} \Omega,
$$

then

$$
\lambda^{\Omega} \leq \mu
$$

Under these conditions we have

THEOREM 3.1: Let 0 be a uniformizer for $F$ whose domain contains 0 and let $u=\log \lambda_{s}$. If $u(0)>-\infty$, then

$$
r^{-2}[m(r ; u)-u(0)] \geq\left[\lambda_{p}(0)\right]^{2}
$$

for sufficiently small positive $r . \lambda$ is an $S-K$ metric.

In order to establish Theorem 3.1, we shall use results concerning the 
solvability of the equation

$$
\Delta u=4 e^{2 u}
$$

exactly as we should use the Poisson integral and its properties to treat the counterpart of Theorem 3.1 for subharmonic functions. An account of existence theorems for the cognate equation

$$
\Delta u=e^{u}
$$

including a report on Bieberbach's investigations [2], is to be found in $[4, \mathrm{pp}$. 286-287]. Since the special results that we shall want concerning (3.1) are rapidly developed, for the sake of completeness we shall establish them.

4. Given a region $\Omega$ in the finite plane. It is immediate that if $\boldsymbol{u}_{1}$ and $\boldsymbol{u}_{2}$ are both continuous and take finite values on $\bar{\Omega}$ [the closure being taken in the sense of the topology of the extended plane], satisfy (3.1) in $\Omega$, and coincide on $\operatorname{fr} \Omega$, then $\boldsymbol{u}_{1}=\boldsymbol{u}_{2}$. It suffices to note that if $\boldsymbol{u}_{1}-\boldsymbol{u}_{2}$ is positive anywhere, it attains a positive maximum at a point $a \in \Omega$. On applying (3.1) we conclude that the Laplacian of $u_{1}-u_{2}$ is positive at $a$. However, since $u_{1}-u_{2}$ has an absolute maximum at $a$, its Laplacian is not positive at $a$. Contradiction. Thus $u_{1}-u_{2} \leq 0$. Similarly $u_{2}-u_{1} \leq 0$. Consequently $u_{1}=u_{2}$.

It is to be observed that all the maximum principles developed for $S \cdot K$ metrics and their specializations have proofs hinging on the fact that at a point of relative maximum the (upper generalized) Laplacian is not positive.

We now establish a very limited existence theorem for (3.1). Later [\$16] we shall see how Perron methods will permit us to obtain global existence theorems adequate for applications. In this paper there will be no concern for refinements. Suppose then that $\Delta(0 ; r)=\{|z|<r\}$ and that $f$ is a given finite, real continuous function on $C(0 ; r)=\{|z|=r\}$. Let $g(z, \zeta)$ denote Green's function for $\Delta(0 ; r)$ relative to the Laplace equation, i.e.

$$
g(z, \zeta)=\log \left|\frac{r^{2}-z \bar{\zeta}}{r(z-\zeta)}\right|
$$

Let

$$
A(r)=\max _{|z|<r} \iint_{\Delta(0 ; r)} g(z, \zeta) d S_{\zeta}
$$

the integration being with respect to euclidean area. It is easily seen that 
$\lim _{r \rightarrow 0} A(r)=0$. Let $B=\max \exp \circ(2 f)$. We have

Theorem 4.1: If

$$
4 \pi^{-1} A(r) B<1
$$

there exists a unique continuous function $v$ on $\overline{\Delta(0 ; r)}$ which is of class $C^{\prime \prime}$ in $\Delta(0 ; r)$, reduces to $f$ on $C(0 ; r)$, and satisfies $(3.1)$ in $\Delta(0 ; r)$.

We establish this result as follows. Given $v$ continuous on $\overline{\Delta(0 ; r)}$, we denote

$$
(2 \pi)^{-1} \iint_{\Delta(0 ; r)} g(z, \zeta) v(\zeta) d S_{\zeta}
$$

by $T[v]$. Now let $h$ denote the solution of the Dirichlet problem (relative to the Laplace equation) with boundary function $f$. If $v$ is a function meeting the imposed conditions, it satisfies

$$
v=h-T\left[4 e^{2 v}\right] .
$$

This suggests the introduction of a sequence of approximants defined as follows :

$$
\left\{\begin{array}{l}
v_{1}=h, \\
v_{n+1}=h-T\left[4 e^{2 v_{n}}\right], n=1,2, \ldots
\end{array}\right.
$$

It follows from (4.3) and

$$
v_{n+2}-v_{n+1}=-T\left[4\left(e^{2 v_{n+1}}-e^{2 v_{n}}\right)\right], n=1,2, \ldots
$$

that with $M_{n}=\max \left|v_{n+1}-v_{n}\right|$,

$$
M_{n+1} \leq 4 \pi^{-1} A(r) B M_{n}
$$

It is immediate by the Weierstrass $M$-test that $\left(v_{n}\right)_{1}^{\infty}$ converges uniformly in $\overline{\Delta(0 ; r)}$. Let $v=\lim v_{n}$. Then $v$ satisfies (4.1). It now follows from standard potential-theoretic results that $v$ fulfills the imposed conditions. We refer to theorems concerning differentiability properties of logarithmic potentials and the Poisson equation. Cf. [4, pp. 228-230].

We note that for $0<t<r, m(t)=m(t ; v)$ satisfies the differential inequality

$$
m^{\prime \prime}(t)+t^{-1} m^{\prime}(t) \geq 4 e^{2 m(t)} \geq 4 e^{2 v(0)} .
$$

It follows that

$$
m^{\prime}(t) \geq 2 e^{2 v(0)} t, 0 \leq t<r .
$$


Hence

$$
m(r)-v(0) \geq e^{2 v(0)} r^{2}
$$

5. Proof of Theorem 3.1. Let $B(r)$ denote $\max _{|z|=r}\left[\lambda_{p}(z)\right]^{2}$. It is assumed that $\overline{\Delta(0 ; r)}$ lies in the domain of $\varphi$. We consider $r_{0}>0$ so small that $4 \pi^{-1} A\left(r_{0}\right) B\left(r_{0}\right)<1$ and that $\varphi\left[\Delta\left(0 ; r_{0}\right)\right]$ lies in the neighborhood $V$ associated with $\varphi(0)$ in $\S 3$. For given $r, 0<r \leq r_{0}$, let $v_{f}$ denote the solution of (3.1) whose existence is asserted in Theorem $4.1, f$ being a continuous function satisfying the condition

$$
u\left(r e^{i \theta}\right) \leq f\left(r e^{i \theta}\right) \leq \frac{1}{2} \log B(r) .
$$

It follows from the condition imposed on $\lambda$ that

$$
u(z) \leq v_{f}(z), \quad|z| \leq r .
$$

Consequently by (4.5) we infer that

$$
m\left(r ; v_{f}\right)-u(0) \geq e^{2 u(0)} r^{2}
$$

Since $u$ is upper semi-continuous, there exists a monotone non-increasing sequence $\left(f_{n}\right)$ of functions continuous on $C(0 ; r)$ which tends pointwise to $u$ on $C(0 ; r)$, each $f_{n}$ satisfying the condition (5.1) imposed on $f$. Hence

$$
m(r ; u)-u(0) \geq e^{2 u(0)} r^{2}, \quad 0<r \leq r_{0} .
$$

Theorem 3.1 follows. We remark that (5.2) is stronger than (1.4). It will follow from subsequent developments [§ 16] that (5.2) holds for all $r$ such that $\overline{\Delta(0 ; r)}$ is contained in the domain of $\varphi$.

6. In Ahlfors's paper there are given criteria guaranteeing that a given conformal metric on $\Delta(0 ; 1)$ is dominated by the hyperbolic metric $\left(1-|z|^{2}\right)^{-1}|d z|$. Actually, the conditions in question considered from our present point of view are simply sufficient conditions for a conformal metric to be an $S-K$ metric. Following the labelling of Ahlfors's paper $[1 ;$ pp. $360-361]$, we introduce the following two definitions:

A 1. A conformal metric $\lambda$ will be said to satisfy the condition A 1 at a point $p \in F$ provided that $\lambda$ does not vanish at $p$ and that for some uniformizer $\varphi . \varphi(0)=p$, there exists a regular arc $\gamma$ lying in the domain of $\varphi, \gamma(0)=0$, which satisfies 


$$
\limsup _{t \downarrow 0} \frac{u \circ \gamma(t)-u(0)}{|\gamma(t)|}+\limsup _{t \downarrow 0} \frac{u \circ \gamma(-t)-u(0)}{|\gamma(-t)|}>0,
$$

where $u=\log \lambda_{4}$.

A 2. A conformal metric $\lambda$ will be said to satisfy the condition A2 at a point $p \in F$ provided that for some uniformizer $\varphi, \varphi(0)=p$, there exists a $C^{\prime \prime}$ function $v$ that has the same domain as $\varphi$ and satisfies: $\Delta v \geq 4 e^{2 v}, v \leq u=\log \lambda_{q}$, $v(0)=u(0)$.

The theorem A 2 of Ahlfors asserts (essentially) that a continuous conformal metric $\lambda$ on $\Delta(0 ; 1)$ which satisfies the condition $\mathrm{A} 2$ at each point is dominated by the hyperbolic metric. Since the differential inequality (4.5) holds for $v$ of A 2 when $r$ is small, we conclude that an upper semi-continuous metric satisfying the condition $\mathrm{A} 2$ at $p$ also satisfies the second condition imposed on an $S-K$ metric for each $\varphi, \varphi(0)=p$. The theorem A 1 of Ahlfors states that a continuous conformal metric $\lambda$ on $\Delta(0 ; 1)$ is dominated by the hyperbolic metric if for each point $z$ where $u=\log \lambda_{c}$ is finite ( $\varepsilon=$ identity map), either there exists a neighborhood of $z$ in which $u$ is $C^{\prime \prime}$ and satisfies $\Delta u \geq 4 e^{2 u}$ or else $u$ satisfies at $z$ a condition involving directional derivatives which implies that $i$ satisfies $\mathrm{A} 1$ at $z$. It is to be observed that the criteria of these theorems are given from the point of view of the immediate applications of the cited paper. We shall see that basically the theorems A 1 and A 2 of Ahlfors give sufficient conditions for a conformal metric to be an $S$ - $K$ metric.

It will be convenient to introduce one more condition of a local character.

B. An upper semi-continuous conformal metric $\lambda$ will be said to satisfy the condition $\mathrm{B}$ at $p$ provided that either $\lambda$ vanishes at $p$ or else for some uniformizer $\varphi, \varphi(0)=p$,

$$
\limsup _{r \rightarrow 0} r^{-2}[m(r ; u)-u(0)] \geq\left[\lambda_{p}(0)\right]^{2}, u=\log \lambda_{p} .
$$

It is to be observed that the condition A 2 implies the condition $\mathrm{B}$.

We now give two sufficient conditions for a conformal metric to be an $S-K$ metric.

THEOREM 6.1: (a) An upper semi-continuous conformal metric $\lambda$ on $F$ is an $S-K$ metric if $\lambda$ satisfies either the condition A 1 or $\mathrm{B}$ at each $p$ where $\lambda$ does not vanish.

(b) A continuous conformal metric $\lambda$ on $F$ is an $S-K$ metric if the condition 
$B$ is fulfilled at each point of the set where $\lambda$ does not vanish save on an arbitrary subset $E$ of zero capacity.

Proof. (a) It suffices to verify that the hypotheses of Theorem 3.1 are satisfied. We consider such $\mu$ and $\Omega$ and suppose that $\lambda^{s} / \mu$ attains a value greater than one at some point of $\Omega$. It follows that $\lambda / \mu$ has an absolute maximum greater than one attained at say $a \in \Omega$. Hence $\lambda$ cannot satisfy the condition $\mathrm{A} 1$ at $a$. The condition B must be satisfied at $a$. With $\varphi$ a uniformizer for $\Omega$ satisfying $\varphi(0)=a, u=\log \lambda_{p}, v=\log \mu_{p}, w=u-v$,

$$
\limsup _{r \rightarrow 0} r^{-2}[m(r ; w)-w(0)] \geq\left[\lambda_{p}(0)\right]^{2}-\left[\mu_{p}(0)\right]^{2}>0 .
$$

On the other hand, $m(r ; w) \leq w(0)$ for $r$ sufficiently small since $\lambda^{\Omega} / \mu$ attains its maximum at $a$. The contradiction is apparent. (a) follows.

(b) Given $p \in F$. Take $V$ as a relatively compact region satisfying: $p \in V \subset \bar{V} \neq F$. Consider an admitted pair $(\mu, \Omega)$. Let $S$ denote a negative subharmonic function $(\neq-\infty)$ on $\Omega$ which takes the value $-\infty$ at each point of $E \cap \Omega$. Let $\eta$ denote a positive number. Then

$$
e^{\eta S}\left(\lambda^{\Omega} / \mu\right) \leq 1
$$

Otherwise, the left hand side of (6.3) attains a maximum $>1$ at a point $a \in \Omega$. We note that $\lambda$ satisfies the condition B at $a$. Let $\varphi$ be a uniformizer for $\Omega$, $\varphi(0)=a$, such that $(6.2)$ is satisfied. Let $v=\log \mu_{\rho}, s=S \circ \varphi, w=u-v+\eta s$. Then $w$ has a positive maximum at 0 , so that

$$
\limsup _{r \rightarrow 0} r^{-2}[m(r ; w)-w(0)] \leq 0 .
$$

On the other hand, from the subharmonicity of $s$,

$$
\begin{aligned}
m(r ; w)-w(0) & =[m(r ; u)-u(0)]+\eta[m(r ; s)-s(0)]-[m(r ; v)-v(0)] \\
& \geq[m(r ; u)-u(0)]-[m(r ; v)-v(0)] .
\end{aligned}
$$

Consequently,

$$
\begin{aligned}
\limsup _{r \rightarrow 0} r^{-2}[m(r ; w)-w(0)] & \geq\left[\lambda_{p}(0)\right]^{2}-\left[\mu_{p}(0)\right]^{2} \\
& \geq\left[\lambda_{p}(0)\right]^{2} e^{2 r s(0)}-\left[\mu_{p}(0)\right]^{2}>0 .
\end{aligned}
$$

Contradiction. Hence $(6.3)$ holds. On letting $\eta \rightarrow 0$, we see that $\lambda^{\Omega} / \mu \leq 1$ thanks to the continuity of $\lambda$. (b) follows.

It is worth remarking that (b) has a "Phragmén-Lindelöf" character. 
7. In pursuing analogies with the theory of subharmonic functions, we are led to ask whether an $S \cdot K$ metric $\lambda$ that is dominated by a $C^{\prime \prime}$ metric $\mu$ of constant eurvature -4 is equal to $\mu$ if $\lambda / \mu$ takes the value 1 somewhere. That this is actually so will be established with the aid of the following lemma.

Lemma 7.1: Let $u_{1}$ and $u_{2}$ denote $C^{\prime \prime}$ real solutions of $\Delta u=4 e^{2 u}$ on $\Delta\left(0 ; r_{n}\right)$. If $u_{1} \geq u_{2}$ and $u_{1}(0)=u_{2}(0)$, then $u_{1}=u_{2}$.

Proof: Let $v=u_{1}-u_{2}$. Given $r, 0<r<r_{0}$, let $h_{k}$ denote the solution of the Dirichlet problem (relative to the Laplace equation) for $\Delta(0 ; r)$ which agrees with $u_{k}$ on $C(0 ; r), k=1,2$. By $(4.2)$ we have

$$
\frac{2}{\pi} \iint_{|\zeta| \leqslant} \log \frac{r}{|\zeta|}\left[e^{2 u_{1}(\zeta)}-e^{2 u_{2}(\zeta)}\right] d S_{\zeta}=h_{1}(0)-h_{2}(0)
$$

Consequently,

$$
m(r ; v) \leq 8 B \int_{0}^{r}\left(\log \frac{r}{t}\right) m(t) t d t
$$

for $0<r \leq r_{1}<r_{0}$, where $B=\frac{\max }{\Delta\left(0 ; r_{1}\right)} e^{2 u_{1}}$.

We have

$$
m(r) \leq 8 B m(r) \int_{0}^{r}\left(\log \frac{r}{t}\right) t d t
$$

It follows that $v(z)=0$ for $z$ sufficiently small. The proof of the lemma now follows from the connectedness of $\Delta\left(0 ; r_{0}\right)$ and the fact that the set where $v=0$ and the set where $v>0$ are both open. The proof that $\{v=0\}$ is open parallels the argument just given.

Theorem 7.1: Let $\lambda$ denote an $S-K$ metric and let $\mu$ denote a $C^{\prime \prime}$ metric of constant curvature -4 on a Riemann surface $F$. If $\lambda / \mu$ has maximum value one, then $\lambda=\mu$.

Proof: Suppose that $\lambda \neq \mu$ : By the upper semi-continuity of $\lambda / \mu$, the set $O$ where $\lambda / \mu$ takes values less than one is open. It is not empty and is a proper subset of $F$. Hence there exists a point $a \in \operatorname{fr} O$. At $a$ the value of $\lambda / \mu$ is 1 . Let $\varphi$ denote a uniformizer, $\varphi(0)=a$, let $u=\log \lambda_{\text {; }}$, and let $v=\log \mu_{\vec{p}}$. For $r(>0)$ sufficiently small, the condition (4.1) is fulfilled if $B$ is replaced by $\max _{|z|=r} \exp \circ(2 v)$. We fix such an $r$ for which $u\left(r e^{i \theta}\right)-v\left(r e^{i \theta}\right) \neq 0$. Let $w$ denote a continuous function on $\overline{\Delta(0 ; r)}$ which satisfies $\Delta w=4 e^{2 w}$ in $\Delta(0 ; r), u\left(r e^{i \theta}\right)$ 
$\leq u\left(r e^{i \theta}\right) \leq v\left(r e^{i \theta}\right)$, and the condition that $w\left(r e^{i \theta}\right) \neq v\left(r e^{i \theta}\right)$. Since $w(0)=v(0)$, by Lemma 7.1 we have $w(z)=v(z),|z|<r$. Hence $w(z)=v(z),|z| \leq r$. Contradiction. Theorem 7.1 follows.

8. Induced metric. Let $f$ denote a conformal map of a Riemann surface $F$ into a Riemann surface $G$ and let $\lambda$ denote a conformal metric on $G$. Then there exists a unique conformal metric $\mu$ on $F$ satisfying the following conditions: if $\sigma$ and $\tau$ are uniformizers for $F$ and $G$ respectively, and if further the image of $f^{\circ} \sigma$ is contained in the image of $\tau$, then

$$
\mu_{3}=\lambda_{\tau} c \theta\left|\theta^{\prime}\right|
$$

where $\theta=\tau^{-1} \circ(f \circ \sigma)$. We term $\mu$ the conformal metric on $F$ induced from $\lambda$ by $f$ and denote it by $[\lambda, f]$. The following properties of induced metrics are readily verified.

(a) If $\lambda_{1} \leq \lambda_{2}$, then $\left[\lambda_{1}, f\right] \leq\left[\lambda_{2}, f\right]$.

(b) If $\mu$ is a conformal metric on a Riemann surface $H, g$ is a conformal map of $G$ into $H$, and $f$ is as above, then

$$
[\mu, g \circ f]=[[\mu, g], f] .
$$

(c) If $f$ is locally simple, $f(F)=G$, and $\left[\lambda_{1}, f\right] \leq\left[\lambda_{2}, f\right]$, then $\lambda_{1} \leq \lambda_{2}$.

(d) If $\lambda$ is $S-K$, then so is $[\lambda, f]$.

(e) Let $f$ be a covering of $G$ and let $F$ be simply-connected. If $\mu$ is a conformal metric on $F$ satisfying $[\mu, \alpha]=\mu$ for each conformal automorphism $\alpha$ leaving $f$ fixed, then there exists a unique conformal metric $\lambda$ on $G$ satisfying $\eta=[\lambda, f]$. If $\mu$ is an $S-K$ metric, then so is $\lambda$.

9. Let $\lambda$ denote an $S$ - $K$ metric on $F$ and let $f$ denote a conformal universal covering of $F$ the domain of which is one of the standard simply-connected regions: $\Delta(0 ; 1)$, the finite plane, the extended plane. Let $\mu=[\lambda, f]$. Let $\iota$ denote the identity map on $\Delta(0 ; 1)$ when the domain of $f$ is $\Delta(0 ; 1)$, and otherwise let c denote the identity map of the finite plane onto itself. It follows from Theorem 2.1 that

$$
\mu(z) \leq \frac{r}{r^{2}-|z|^{2}} \quad|z|<r,
$$

where $0<r<1$ when the domain of $f$ is $\Delta(0 ; 1)$ and otherwise $r$ is finite and positive. Consequently, we obtain 
THEOREM 9.1: If a Riemann surface $F$ possesses an $S-K$ metric that is not identically zero, then the domain of a conformal universal covering of $F$ is hyperbòlic.

Further, when the domain of $f$ is $\Delta(0 ; 1),(9.1)$ yields

$$
\mu_{c}(z) \leq\left(1-|z|^{2}\right)^{-1}, \quad|z|<1 .
$$

There is a unique conformal metric $r$ on $F$ such that

$$
[\gamma, f]_{\iota}(z)=\left(1-|z|^{2}\right)^{-1}, \quad|z|<1,
$$

as follows from $\S 8(\mathrm{e})$. Further, if $f_{1}$ and $f_{2}$ are conformal universal coverings of $F$ with domain $\Delta(0 ; 1)$ and if

$$
\left[\gamma_{k}, f_{k}\right]_{c}(z)=\left(1-|z|^{2}\right)^{-1}, \quad|z|<1,
$$

then, as follows easily from $\S 8(\mathrm{~b}), \cdot(\mathrm{e}), \gamma_{1}=\gamma_{2}$. The common conformal metric associated with the conformal universal coverings of $F$ is termed the hyperbolic metric. It will be denoted by $\gamma$. We note that $\gamma$ has constant curvature -4 . From (9.2) and $\S 8(c)$, we obtain

Theorem 9.2: Let $\lambda$ denote an $S-K$ metric on a Riemann surface $F$ and let the domain of a conformal universal covering of $F$ be hyperbolic. Then

$$
\lambda \leq r .
$$

This theorem generalizes the lemma of Schwarz-Pick-Ahlfors [1]. Two comments should be made. First, the inequality (9.1) and its consequence (9.2) are the essential steps in the argument of Ahlfors for the case of the unit disk. Second, the inequality (9.4) shows that $\gamma$ is the maximal $C^{\prime \prime}$ metric of constant curvature -4 on $F$.

10. General properties of $S-K$ metrics. The first two properties, (a) and (b) below, are easily verified.

(a) If $\lambda^{1}$ and $\lambda^{2}$ are $S$ - $K$ metrics on $F$, then so is $\max \left\{\lambda^{1}, \lambda^{2}\right\}$.

(b) If $\lambda$ is an $S-K$ metric on $F$ and $s$ is a negative subharmonic function on $F$, then (expos) $\lambda$ is an $S-K$ metric on $F$.

The essential part of the proof of (b) is already implicit in the proof of Theorem 6.1 (b). It suffices to employ the mean-value property for subharmonic functions and the fact that $s<0$. 
Sequences. It is immediate how notions of "pointwise convergence", uniform convergence", etc. are introduced for sequences of conformal metrics by reference to the behavior of the functions that the metrics assign to uniformizers. The following holds:

(c) If $\left(\lambda^{n}\right)$ is a monotone non-increasing sequence of $S-K$ metrics on a Riemann surface $F$, then the pointwise limit is also an $S-K$ metric on $F$. If $\left(\lambda^{n}\right)$ is non-decreasing and $\lambda$ is its limit, then the "regularization" $\bar{\lambda}$ of $\lambda$ given by $\sigma \rightarrow \bar{\lambda}_{\sigma}, \bar{\lambda}_{\sigma}$ being the upper limit function of $\lambda_{\sigma}$ in the sense of Caratheodory, is an $S-K$ metric ( $\lambda$ need not be upper semi-continuous).

Let $\lambda^{1}, \ldots, \lambda^{n}$ denote $n$ given conformal metrics on a Riemann surface $F$, and let $\eta_{1}, \ldots, \eta_{n}$ denote $n$ positive numbers, $\sum_{k=1}^{n} \eta_{k}=1$. Let $\lambda$ be defined on $S$ by

$$
\lambda_{\sigma}=\prod_{k=1}^{n}\left(\lambda_{\sigma}^{k}\right)^{\eta_{k}}
$$

It is immediate that $\lambda$ is a conformal metric on $F$. It will be denoted by $\prod_{1}^{n}\left(\lambda^{k}\right)^{\eta / k}$ (weighted geometric mean of $\lambda^{1}, \ldots, \lambda^{n}$ ).

(d) If $\lambda^{1}, \ldots, \lambda^{n}$ are $S-K$ metrics, then so is $\lambda=\prod_{1}^{n}\left(\lambda^{k}\right)^{\eta_{k}}$.

The upper semi-continuity property of $\lambda$ is immediate. The mean-value property of $\lambda$ at a point where $\lambda$ does not vanish is readily concluded with the aid of the inequality of the arithmetic and geometric means. In fact, if $\lambda$ does not vanish at $a$, let $\varphi$ denote a uniformizer, $\varphi(0)=a$, let $u_{k}=\log \lambda_{p}^{k}$, and let $v=\sum \eta_{k} u_{k}$. Then for $r>0$ sufficiently small

$$
\begin{aligned}
m(r ; v)-v(0) & =\sum \eta_{k}\left[m\left(r ; u_{k}\right)-u_{k}(0)\right] \\
& \geq \sum \eta_{k} r^{2} e^{2 u_{k}(0)} \\
& \geq r^{2} e^{2 v(0)} .
\end{aligned}
$$

Another way of constructing an $S \cdot K$ metric arises from the property (e) that will be stated below. Let $\mu$ denote a euclidean metric on $F$. By this we mean that $\mu$ is continuous and that for each $\sigma \in S, \log \mu_{0}$ is harmonic save for a set of zero capacity at each point of which $\mu_{3}$ vanishes. Suppose that $\lambda$ is an $S \cdot K$ metric on $F$ which satisfies the condition that there exists a positive number $A$ such that

$$
\lambda \geq A \mu \text {. }
$$


Then

(e) For each $\eta, 0<\eta<1$, there exists a positive constant $B$ such that

$$
B \lambda^{1-\eta} \mu^{\eta}
$$

is an $S \cdot K$ metric on $F$.

To see that this is so, it suffices to verify the mean-value property at a point $a \in F$ at which neither $\lambda$ nor $\mu$ vanishes. Let $\varphi$ denote a uniformizer, $\varphi(0)=a$; let $u=\log \lambda_{p}, v=\log \mu_{p}$, and $w=(1-\eta) u+\eta v$. For $r>0$ sufficiently small

$$
\begin{aligned}
m(r ; w)-w(0) & \geq(1-\eta) r^{2} e^{2 u(0)} \\
& =(1-\eta) r^{2} e^{2 w(0)} e^{2 \eta[u(0)-v(0)]} \\
& \geq(1-\eta) r^{2} e^{2 w(0)} A^{2 \eta}
\end{aligned}
$$

It is now immediate that (e) follows when $B$ satisfies

$$
B^{2} \leq(1-\eta) A^{2 \eta}
$$

11. Sequences of metrics of constant curvature -4 . The basic result that we shall establish here is

TheOREM 11.1: The family of the $C^{\prime \prime}$ conformal metrics of constant curvature -4 on a given Riemann surface $F$ is normal in the sense of Montel. The limit of a convergent sequence of such metrics is either a metric of the same type or else is identically zero.

The proof of the theorem will be based upon the following lemma.

LEMMA 11.1: Let $\left(u_{n}\right)_{1}^{\infty}$ denote a sequence of real-valued functions, each belonging to $C^{\prime \prime}[\Delta(0 ; 1)]$ and satisfying

$$
\Delta u=4 e^{2 u} .
$$

If $u_{n}(0) \rightarrow-\infty$, then $u_{n} \rightarrow-\infty$ uniformly in $\Delta(0 ; 1)$. If $\liminf _{n \rightarrow \infty} u_{n}(0)>-\infty$, then $\left(u_{n}\right)_{1}^{\infty}$ is equicontinuous in $\Delta(0 ; 1)$ and is bounded on each compact subset of $\Delta(0 ; 1)$. If $\left(u_{n}\right)_{1}^{\infty}$ is pointwise convergent to a finite-valued function $u$, then $u \in C^{\prime \prime}[\Delta(0 ; 1)]$ and satisfies (11.1).

We remark that "uniformly in" = "uniformly on each compact subset of" and "equicontinuous in" = "equicontinuous on each compact subset of".

The proof of the lemma will be based on the observation [Schwarz-Pick- 
Ahlfors lemma for $\Delta(0 ; 1)]$ that

$$
u_{n}(z) \leq-\log \left(1-|z|^{2}\right), \quad|z|<1 ; n=1,2, \ldots,
$$

and the use of potential-theoretic identities satisfied by the $u_{n}$. Let us fix $r, 0<r<1$, and let $h_{n}$ denote the least harmonic majorant of the restriction of $u_{n}$ to $\Delta(0 ; r)$. Using the notation of $\S 4$ we have

$$
u_{n}(z)=-p_{n}(z)+h_{n}(z), \quad|z|<r,
$$

where $p_{n}=T\left[4 \exp \circ\left(2 u_{n}\right)\right]$.

If $u_{n}(0) \rightarrow-\infty$, then, as we see from (11.2) and (11.3), $h_{n}(0) \rightarrow-\infty$. Since $h_{n}(z) \leq-\log \left(1-r^{2}\right),|z|<r$, it follows that $h_{n} \rightarrow-\infty$ uniformly in $\Delta(0 ; \boldsymbol{r})$. Hence $u_{n} \rightarrow-\infty$ uniformly in $\Delta(0 ; r)$. The first assertion of the lemma follows.

If $\liminf _{n \rightarrow \infty} u_{n}(0)>-\infty$, then $\left(h_{n}\right)_{1}^{\infty}$ is equicontinuous in $\Delta(0 ; r)$ and is bounded on each compact subset of $\Delta(0 ; r)$. Since

$$
\left|p_{n}\left(z_{1}\right)-p_{n}\left(z_{2}\right)\right| \leq \frac{2}{\pi\left(1-r^{2}\right)^{2}} \iint_{|\zeta|<r}\left|g\left(z_{1}, \zeta\right)-g\left(z_{2}, \zeta\right)\right| d S,
$$

$\left|z_{k}\right|<r$, where $g$ is Green's function for $\Delta(0 ; r)$, we infer that $\left(p_{n}\right)_{1}^{\infty}$ is equicontinuous in $\Delta(0 ; r)$. Hence $\left(u_{n}\right)$ is equicontinuous in $\Delta(0 ; 1)$. It is - also bounded on each compact subset of $\Delta(0 ; 1)$.

It now follows from (11.3) that if $u_{n} \rightarrow u$ pointwise in $\Delta(0 ; 1)$, then $u$ satisfies

$$
u(z)=-T[4 \exp \circ(2 u)]+h(z), \quad|z|<r,
$$

where $h$ is the harmonic function on $\Delta(0 ; r)$ with continuous boundary values equal to $u$. From (11.5) we conclude that $u \in C^{\prime \prime}[\Delta(0 ; 1)]$ and satisfies (11.1). The proof of the lemma is complete.

It is now easy to see how Lemma 11.1 leads us to the normality properties of the family of $C^{\prime \prime}$ conformal metrics of constant curvature -4 on a given Riemann surface. It is well to state that by the normality of the family we mean that for each sequence $\left(\lambda^{n}\right)$ of such metrics there exists a subsequence $\left(\lambda^{m(n)}\right)$ such that for each $\sigma \in S$ the sequence $\left(\lambda_{\sigma}^{m(n)}\right)$ is uniformly convergent in the domain of $\sigma$. Theorem 11.1 is now readily concluded on introducing a countable family of uniformizers, each with domain $\Delta(0 ; 1)$ whose images cover $F$. It suffices to apply Lemma 11.1 and the selection principle to establish the first 
assertion and to use a standard connectedness argument to establish the second assertion.

We remark that a sequence $\left(\lambda^{n}\right)$ of $C^{\prime \prime}$ conformal metrics of constant curvature -4 converges uniformly in $F$ when it converges pointwise, in particular, when $\left(\lambda^{n}\right)$ is monotone. Further, we mention the following result that depends upon the fact that a $C^{\prime \prime}$ solution of (11.1) is actually real analytic [4, pp. 339-342], a property that we shall not use elsewhere in this paper: If $a$ sequence $\left(\lambda^{n}\right)$ of $C^{\prime \prime}$ (alias real analytic) conformal metrics of constant curvature -4 converges pointwise on a non-empty open set, then $\left(\lambda^{n}\right)$ converges on $F$.

12. Modification. One of our objects is to apply the method of Perron to the study of $S \cdot K$ metrics and their majorants of constant curvature -4 . A tool that we shall want to develop is the modification of an $S-K$ metric. It is the counterpart of the Poisson modification of a subharmonic function in the original Perron theory. We first prove

TheOREM 12.1: Let $\lambda$ denote an $S-K$ metric on $F$ and let $\varphi$ denote a uniformizer the domain of which contains $\overline{\triangle(0 ; R)}$. Then there is at most one $S-K$ metric $\mu$ on $F$ satisfying:

(a) $\lambda^{E}=\mu^{E}$, where $E=F-\varphi[\Delta(0 ; R)]$;

(b) $u$, the restriction of $\log \mu_{p}$ to $\Delta(0 ; R)$, belongs to $C^{\prime \prime}[\Delta(0 ; R)]$ and satisfies $\Delta u=4 e^{2 u}$.

Proof: Let $\nu$ also denote an $S$ - $K$ metric on $F$ meeting the conditions imposed on $\mu$, and let $w$ denote the restriction of $\log \nu_{\varphi}$ to $\Delta(0 ; R)$. To establish the theorem it suffices to show that $u=w$. Given $r, 0<r<R$, we have

$$
u(z)=-T[4 \exp \circ(2 u)]+h(z), \quad|z|<r,
$$

where $h$ is the harmonic function on $\Delta(0 ; r)$ given by the Poisson integral for $\Delta(0 ; r)$ with boundary function $u$, as well as a corresponding identity for $w$. We let $r \rightarrow R$ and infer that the counterparts of (12.1) hold for $u$ and $w$ on $\Delta(0 ; R)$, as may be concluded by using the subharmonicity of $\log \mu_{\varphi}$ and $\log \nu_{p}$. Hence $u-w$ vanishes continuously at each point of $\{|z|=R\}$. Now $|u-w|$ is subharmonic and hence vanishes. The theorem follows.

We shall agree to term an $S$ - $K$ metric $\mu$ meeting the conditions of Theorem 12.1 the $(\varphi, R)$ modification of $\lambda$. More simply, we shall say that an $S$ - $K$ metric 
is a modification of $\lambda$ provided that it is the $(\varphi, R)$ modification of $\lambda$ for some $(\varphi, R)$.

We now establish an existence theorem for modifications.

TheOREм 12.2: Let $\varphi$ denote a uniformizer for $F$, the domain of which contains 0 . There exists $r_{0}>0$ for which $\overline{\Delta\left(0 ; r_{0}\right)}$ is contained in the domain of $\varphi$ such that each $S-K$ metric $\lambda$ on $F$ which does not vanish everywhere admits $a(\varphi, r)$ modification, $0<r \leq r_{0}$.

Proof: Given an admitted $\lambda$, from the subharmonicity of the $\log \lambda_{\sigma}$, we conclude that cap $\{\lambda=0\}=0$. We fix $R>0$ such that $\Delta(0 ; R)$ is contained in the domain of $\varphi$. For $|z|<R$ and each $\lambda$ we have

$$
\lambda_{\varphi}(z) \leq R\left(R^{2}-|z|^{2}\right)^{-1} .
$$

We now fix $r_{0}, 0<r_{0}<R$, such that the condition (4.1) is fulfilled with

$$
B=\left(\frac{R}{R^{2}-r_{0}^{2}}\right)^{2}
$$

and $r=r_{0}$. We now consider a given admitted $\lambda$ and let $u=\log \lambda_{\varphi}$. Let $\left(f_{n}\right)$ denote a monotone non-increasing sequence of continuous functions on $\{|z|=r\}$, $0<r \leq r_{0}$, which tends pointwise to $u$ on $\{|z|=r\}$, the sequence being so chosen that $\max f_{1} \leq(\log B) / 2$. Let $v_{n}$ denote the solution of the boundary value problem for $\Delta v=4 e^{2 v}$ on $\Delta(0 ; R)$ with boundary function $f_{n}$. The sequence $\left(v_{n}\right)$ is monotone non-increasing and tends to a function $v \in C^{\prime \prime}[\Delta(0 ; r)]$ satisfying: $\Delta v=4 e^{2 v}$ and $v(z) \geq u(z),|z|<r$.

Now let $\mu$ denote the unique conformal metric on $F$ satisfying: (a) $\mu^{F}=\lambda^{E}$, where $E=F-\varphi[\Delta(0 ; r)]$, and (b) $\log \mu_{r}(z)=v(z),|z|<r$. We verify that $\mu$ is an $S$ - $K$ metric and is, in fact, the $(\varphi, r)$ modification of $\lambda$. The upper semicontinuity of $\mu$ at a point not on $\varphi\{|z|=r\}$ is obvious. To show that $\mu$ is upper semi-continuous at each point of $\varphi\{|z|=r\}$ it suffices to show the upper semi-continuity of $\log \mu_{p}$ at each point of $\{|z|=r\}$. But $\lim \sup \log \mu_{p} \leq f_{n}(z)$, $|z|=r, n=1,2, \ldots$ Hence $\lim \sup \log \mu_{p} \leq \log \mu_{p}(z)$. It suffices to check the mean-value property only for points of $\varphi\{|z|=r\}$. Here, just as in the case of subharmonic functions, the desired mean-value property is verified thanks to the fact that $\lambda_{p} \leq \mu_{p}$ and $\lambda_{p}(z)=\mu_{p}(z),|z|=r$.

13. Perron family of $S \cdot K$ metrics. A family $\Phi$ of $S \cdot K$ metrics on $F$ will 
be termed a Perron family provided that the following two conditions hold: (a) If $\lambda, \mu \in \emptyset$, then $\max \{\lambda, \mu\} \in \emptyset$. (b) If $\mu$ is a modification of $\lambda(\neq 0) \in \emptyset$, then $\mu \in \emptyset$. Our main concern in the present section will be to study the upper envelope of a given Perron family and to show that it is a $C^{\prime \prime}$ metric of constant curvature -4 whenever the family contains members that do not vanish every. where. Of course, by the upper envelope of a family $\Psi(\neq \varnothing)$ of conformal metrics on $F$ such that some conformal metric dominates every member, we mean the unique conformal metric $\mu$ on $F$ satisfying: (a) $\lambda \leq \mu, \lambda \in \Psi$, (b) if $\lambda \leq \nu, \lambda \in \Psi$ then $\mu \leq \nu$. Before we turn to this question, let us remark that the set of all $S$ - $K$ metrics on $F$ is a Perron family, and further that if $\Psi$ is an arbitrary family of $S$ - $K$ metrics on $F$, there is a minimal Perron family $\emptyset[\Psi]$ containing $\Psi$, namely the intersection of all Perron families containing $\Psi$.

The fundamental theorem is

Theorem 13.1: If $\Phi$ is a Perron family containing a metric not vanishing everywhere, then the upper envelope of $\Phi$ is a $C^{\prime \prime}$ metric of constant curvature $-4$.

Proof: The proof runs parallel to that for the subharmonic situation, the major variant lying in the use of Theorem 12.2 in place of the corresponding theorem for subharmonic functions. We fix a uniformizer $\varphi$ with domain containing 0 and choose $r(>0$ ) so small that every $S-K$ metric ( $\neq 0)$ on $F$ admits a $(\varphi, r)$ modification. We proceed by showing that $\log \mu$, is $C^{\prime \prime}$ in $\Delta(0 ; r)$ and satisfies there $\Delta u=4 e^{2 u}$, $\mu$ being the upper envelope of $\emptyset$. To that end, let $\left(\lambda^{k}\right)$ denote a monotone non-decreasing sequence of members $(\neq 0)$ of $\Phi$ such that each $\lambda^{k}$ is its own $(\varphi, r)$ modification and $\lim \lambda_{p}^{k}(0)=\mu_{p}(0)$; let $u=\lim _{k \rightarrow \infty} \log \lambda_{\varphi}^{k}$; let $a(\neq 0) \in \Delta(0 ; r)$ and let $\left(\tilde{\lambda}^{k}\right)$ denote a non-decreasing sequence of members of $\Phi$ such that (a) $\lim \tilde{\lambda}_{p}^{k}(a)=\mu_{p}(a)$, (b) $\tilde{\lambda}^{k} \geq \lambda^{k}, k=1,2, \ldots$, (c) each $\tilde{\lambda}^{k}$ is its own $(\varphi, r)$ modification; let $v=\lim \log \tilde{\lambda}_{\varphi}^{k}$. Then $v \geq u$ and $v(0)$ $=u(0)$. It follows from I.emma 7.1 that $v(z)=u(z),|z|<r$. Consequently, $\log \mu_{p}(z)=u(z),|z|<r$. We conclude that $\mu$ is a $C^{\prime \prime}$ conformal metric of constant curvature -4 .

Theorem 13.1 is rich in consequences. We note that if $\nu$ is a $C^{\prime \prime}$ conformal metric of constant curvature -4 , then the family of $S \cdot K$ metrics $\lambda$ satisfying $\lambda \leq \nu$ is a Perron family. We conclude 
THEOREM 13.2: If $\Psi$ is a family of $S-K$ metrics containing a member that does not vanish everywhere, then the upper envelope of $\Phi[\Psi]$ is the least $C^{\prime \prime}$ conformal metric of constant curvature -4 dominating each member of $\Psi$.

Of course, $\Psi$ may reduce to a single element. In this case we have in the upper envelope of $\Phi[\Psi]$ the counterpart of the least harmonic majorant of a subharmonic function.

An application of Theorem 13.2. Suppose that $\lambda$ is an $S-K$ metric on $F$ which does not vanish everywhere. The set where $\lambda$ vanishes is a $G_{\delta}$ of zero capacity. If a Riemann surface admits a non-trivial $S-K$ metric, there exists an $S-K$ metric with the property that the set on which it vanishes is a given $G_{\delta}$ of zero capacity [cf. $\S 15]$. For the present we consider a problem concerning the existence of an $S$ - $K$ metric that is $C^{\prime \prime}$ and of constant curvature -4 on the complement of a closed set $E$ of zero capacity, the behavior on $E$ being restricted in a manner that will be made explicit.

To be precise, let. $\lambda$ denote an $S$ - $K$ metric on $F$. We suppose that for each uniformizer $\varphi$ with domain $\Delta(0 ; 1)$ there exists $r_{\psi}, 0<r_{p}<1$, and a function $P_{p}$ with domain $\Delta\left(0 ; r_{\varphi}\right)$ satisfying: (a) $P_{p}$ is non-negative and is harmonic save at the points of a compact set of zero capacity (possibly empty) at each point of which $P_{p}$ takes the value $+\infty$ and is continuous, (b) $P_{\varphi}$ vanishes conti. nuously on $C\left(0 ; r_{p}\right),(c)$

$$
\log \lambda_{\varphi}(z)+P_{\varphi}(z)+\log \left(1-r_{\varphi}^{2}\right)=O(1),
$$

for $z \in \Delta\left(0 ; r_{p}\right)$ satisfying $P_{\varphi}(z) \neq+\infty$.

The motivation for (13.1) lies in the fact that we wish to impose a fairly simple local behavior on $\lambda$ at the points where it vanishes. The condition (13.1) and the subharmonicity of $\log \lambda_{p}$ imply that the left-hand side of (13.1) is nonpositive. Because of condition (13.1) the set of points at which $\lambda$ vanishes is a closed set $E$ of zero capacity. Let $\Omega=F-E$. We introduce two Perron families on $\Omega$. Let $\Phi_{1}$ be the Perron family generated by $\lambda^{\Omega}$. Let $\Phi_{2}$ denote the family of $S$ - $K$ metrics $\mu$ on $\Omega$ satisfying

$$
0 \geq \log \mu_{\varphi}(z)+P_{\varphi}(z)+\log \left(1-r_{p}^{2}\right)
$$

for $z \in \Delta\left(0 ; r_{p}\right)$ satisfying $P_{\varphi}(z) \neq+\infty, \mu_{p}$ denoting the image of $\mu$ with respect to the restriction of $\varphi$ to $\left\{P_{\rho}(z) \neq+\infty\right\}$. 
Now $\Phi_{2}$ is not empty since $\lambda^{\Omega} \in \Phi_{2}$. The maximum of two members of $\Phi_{2}$ belongs to $\Phi_{2}$. Let $\mu \in \Phi_{2}$ and let $\widetilde{\mu}$ denote a modification of $\mu$. With $\widetilde{\mu}_{\text {, denot- }}$ ing the image of $\tilde{\mu}$ with respect to the restriction of $\varphi$ to $\left\{P_{p}(z) \neq+\infty\right\}$, we have

$$
\widetilde{\mu}_{\rho}(z) \leq \frac{1}{1-|z|^{2}} .
$$

It now follows that (13.2) is satisfied when $\mu$ is replaced by $\tilde{\mu}$. Consequently $\emptyset_{2}$ is a Perron family.

Let $\lambda_{k}$ denote the extension of the upper envelope of $\Phi_{k}(k=1,2)$ to $F$ which vanishes on $E$. Then the $\lambda_{k}$ are continuous and satisfy

$$
\lambda \leq \lambda_{1} \leq \lambda_{2}
$$

and (13.2) is satisfied when $\mu$ is replaced by $\lambda_{2}$. On $\Omega$ the $\lambda_{k}$ reduce to $C^{\prime \prime}$ conformal metrics of curvature -4 . It is to be observed that $\lambda_{1}$ and $\lambda_{2}$ may very well be distinct. We are led to the following theorem.

TheOREM 13.3: Let $E$ denote a discrete set $\subset F$ and let $a$ positive number $\alpha(a)$ be assigned to each point $a \in E$. If there exists an $S \cdot K$ metric $\lambda$ on $F$ satisfying: (a) its restriction to $F-E$ is $C^{\prime \prime}$ and of constant curvature - 4, (b)

$$
\log \lambda_{\sigma}(z)-\alpha(a) \log |z|=O(1),
$$

$z$ small, o being a uniformizer, $\sigma(0)=a$, then there is a maximal $S-K$ metric meeting the conditions imposed on $\lambda$.

The proof is easy. Given $\varphi$, we have for some $r, 0<r<1$,

$$
\log \lambda_{p}(z) \leq \log \frac{1}{1-r^{2}}-\delta[\varphi(0)] \log \frac{r}{|z|}, \quad|z|<r,
$$

where $\delta(a)=\alpha(a)$ if $a \in E$ and otherwise $\delta(a)=0$. We take $P_{\phi}$ to be $\delta[\varphi(0)] \log (\boldsymbol{r} /|z|)$. We then see that $\lambda_{2}$ is the maximal $\lambda$ satisfying the imposed conditions.

Theorem 13.3 will play a useful role when we consider function-theoretic questions later.

It is worth remarking that if $0<\beta(a) \leq \alpha(a), a \in E$, then under the hypotheses of Theorem 13.3 there exists an $S-K$ metric meeting the conditions imposed on $\lambda$ with $\beta(a)$ replacing $\alpha(a)$.

14. Metrics with assigned zeros. It is of interest to inquire whether there 
exists on a given Riemann surface $F$ admitting a hyperbolic metric a continuous conformal metric that vanishes at an assigned point $a \in F$, its restriction to $F-\{a\}$ being $C^{\prime \prime}$ and of constant curvature -4 .

The existence of such a metric is very easy to establish when $F$ is hyperbolic (i.e. when Green's function exists). To see this, let $\&$ denote Green's function for $F$ with pole $a \in F$, and let $\alpha$ denote a given positive number. Then

$$
[\exp \circ(-\alpha g)] \gamma
$$

is an $S$ - $K$ metric with a zero of order $\alpha$ at $a, \gamma$ being the hyperbolic metric of $F$. It follows from the consideration of the Perron family $\Phi$ generated by the restriction of (14.1) to $F-\{a\}$ that there exists a continuous conformal metric on $F$ having a zero of order $\alpha$ at $a$ whose restriction to $F-\{a\}$ is $C^{\prime \prime}$ and of constant curvature -4 . This conclusion follows readily on noting that if $\varphi$ satisfies $\varphi(0)=a, \varphi$ being a uniformizer with domain $\Delta(0: 1)$, then

$$
\lambda_{p}(z) \leq \frac{(\alpha+1)|z|^{\alpha}}{1-|z|^{2(\alpha+1)}}, \quad|z|<1, \lambda \in \emptyset .
$$

It is to be observed that even the order of the zero is controlled.

If $F$ is a compact Riemann surface of genus $>1$ then, as we shall see, there do exist admitted conformal metrics vanishing at an assigned point, but the order of the zero is severely restricted in terms of the genus. [The latter remark will become clear in the light of $\$ 21$ where the conformal metrics of Schwarz and Picard are considered.] There also exist admitted conformal metrics when $F$ is a non-compact parabolic Riemann surface, however it is not known to me how far the order of the zero can be controlled in this case.

We start with an obvious lemma that will permit us to construct an $S-K$ metric leading to a conformal metric of the desired type. Let

$$
\mu(\rho)=\frac{\alpha R^{\alpha} \rho^{\alpha-1}}{R^{2 \alpha}-\rho^{2 \alpha}}
$$

where $R>0, \alpha>1,0<\rho<R$. We have

Lemma 14.1: Let $r, \eta$ denote given positive numbers. Then there exist $R>r$ and $\alpha>1$ such that

$$
\mu(r)<\eta, \mu^{\prime}(\boldsymbol{r}) / \mu(\boldsymbol{r})<\eta \text {. }
$$

Suppose now that $\Gamma$ is a Fuchsian or Fuchsoid group operating on $\Delta(0 ; 1)$, 
We allow $I$ to contain elliptic transformations. Let $r, 0<r<1$, be so chosen that the disks $\tau[\overline{\Delta(0 ; r)}], \tau \in \Gamma$, are mutually disjoint if there is no elliptic member of $\Gamma$ with fixed point 0 , but are disjoint or coincide if there is an elliptic member with fixed point zero. We first construct an $S-K$ metric $\lambda$ on $\Delta(0 ; 1)$ invariant with respect to the transformations of $\Gamma$, i.e. satisfying $[\lambda, \tau]=\lambda, \tau \in I$ : To that end, we take

$$
\eta=\frac{\min \{2 r, 1\}}{1-r^{2}}
$$

and choose $R$ and $\alpha$ to satisfy Lemma 14.1 with reference to this value of $\eta$. Let $k=\mu(r)\left(1-r^{2}\right)<1$. Since we are concerned with the unit disk, we may harmlessly use the same notation for a conformal metric and its scale function associated with the identity. We define

$$
\lambda(\tau z)=\mu(|z|)\left|\tau^{\prime}(z)\right|^{-1}, \quad|z| \leq r, \tau \in \Gamma .
$$

This definition is consistent in the case where an elliptic transformation with fixed point zero is present in $\Gamma$. For $z \in \Delta(0 ; 1)-U \tau[\overline{\Delta(0 ; 1)}]$ we define

$$
\lambda(z)=\frac{k}{1-|z|^{2}} .
$$

It is now easily verified that $\lambda$ is an $S-K$ metric on $\Delta(0 ; 1)$ thanks to the choice of $R$ and $\alpha$. Further $\lambda$ has a zero of order $\alpha-1$ at each point of the orbit $\{\tau 0\}_{\tau \in \Gamma}$ and is invariant with respect to the transformations of $\Gamma$. It follows from the results of $\S \mathbf{1 3}$ that there exists a maximal continuous conformal metric $\tilde{\lambda}$ on $\Delta(0 ; 1)$ which has zeros of order $\alpha-1$ at each of the points $\tau 0$ and is such that its restriction to $\Delta(0 ; 1)-\{\tau 0\}_{\tau \in \Gamma}$ is $C^{\prime \prime}$ and has constant curvature -4 . [When we say that $\tilde{\lambda}$ has a zero of order $\alpha-1$ at $a$, we mean $\log \left[\tilde{\lambda}(z)|z-a|^{1-\alpha}\right]=O(1)$ for $z$ near $a$. Cf. however $\S 18$. $]$ For each $\tau \in I$ we have

$$
[\tilde{\lambda}, \tau] \leq \tilde{\lambda} .
$$

From $\S 8$ (a), (b) we conclude that

$$
[\tilde{\lambda}, \tau]=\tilde{\lambda} .
$$

Applying $\S 8$ (e), we conclude, on considering the case where, $\Gamma$ is the group of conformal automorphisms leaving invariant a conformal universal covering of 
a Riemann surface $F$ with domain $\Delta(0 ; 1)$, that there exists a continuous conformal metric on $F$ which vanishes at just one assigned point and elsewhere is $C^{\prime \prime}$ and of constant curvature -4 .

15. The zero set of an $S-K$ metric. The method of $\$ 14$ permits us to characterize the set where an $S$ - $K$ metric vanishes. We have seen that the set is a $G_{\delta}$ of zero capacity when the metric does not vanish identically. We shall now see that if $X$ is a $G_{\delta}$ of zero capacity lying in a Riemann surface $F$ admitting a hyperbolic metric, then there exists an $S$ - $K$ metric on $F$ whose zero set is $X$.

Thanks to a result of Deny [5], if $0<\rho<r<1$ and $E$ is a $G_{\delta}$ of zero capacity lying in $\Delta(0 ; \rho)$, there exists a Green's potential $P$ (relative to $\Delta(0 ; r)$ ) generated by a mass distribution in $\overline{\Delta\left(0 ; \rho_{1}\right)}, \rho<\rho_{1}<r$, whose infinities consist precisely of the points of $E$. Given $\varepsilon>0$, there exist $\eta>0$ and $R>1$ such that the maximum of the outer normal derivative along $C(0 ; r)$ of

$$
z \rightarrow-\eta P(z)+\log \frac{R}{R^{2}-|z|^{2}}
$$

is less than $\varepsilon$. The argument of $\$ 14$ may now be applied to construct an $S-K$ metric on $\Delta(0 ; 1)$ satisfying: (a) it is invariant under the transformations of a given Fuchsian or Fuchsoid group acting on $\Delta(0 ; 1),(\mathrm{b})$ the set on which it vanishes is of the form $U_{\tau \in \Gamma \tau} \tau(E)$ where $E$ is a $G_{\delta}$ of zero capacity contained in a non-euclidean disk of sufficiently small radius and invariant under $\tau \in \Gamma$ leaving the disk fixed. It suffices to consider the case where the non-euclidean disk is of the form $\Delta(0 ; \rho)$ and for some $r, \rho<r<1, \Delta(0 ; r)$ satisfies the condition relative to $\Gamma$ stated in $\S \mathbf{1 4}$. We replace $k$ of $\S 14$ by

$$
\frac{R\left(1-r^{2}\right)}{R^{2}-r^{2}}
$$

and take $\varepsilon=2 r\left(1-r^{2}\right)^{-1}$. We replace $\mu(|z|)$ by

$$
\exp [-\eta P(z)] \frac{R}{R^{2}-|z|^{2}}
$$

in (14.4). Finally, we suppose that $P$ is invariant under the transformations $\tau \in \Gamma$ with fixed point 0 .

If $\Gamma$ has a compact fundamental domain and $E$ is a $G_{\delta}$ in $\Delta(0 ; 1)$, of zero capacity, and invariant under the transformations of $\Gamma$, then there exist $S-K$ 
metrics $\mu_{1}, \ldots, \mu_{n}$, each invariant under the transformations of $\Gamma$, such that the union of the sets on which the $\mu_{k}$ vanish is $E$. It follows that

$$
\prod_{1}^{n} \mu_{k}^{1 / n}
$$

is an $S$ - $K$ metric invariant under the transformations of $r$ and that the set on which it vanishes is $E$.

The case where the fundamental domain of $\Gamma$ is not compact admits a parallel treatment save that here convergence questions arise. They may be handled by the proper use of weighted means. To treat this case, we start by observing that there exists a sequence of $S$ - $K$ metrics $\left(\mu_{n}\right)_{1}^{\infty}$ on $\Delta(0 ; 1)$ having the following properties: (a) each $\mu_{n}$ is invariant under the transformations of $\Gamma$, (b) the union $E$ of the sets where the $\mu_{n}$ vanish is a given $G_{\delta}$ of zero capacity in $\Delta(0 ; 1)$ invariant under the transformations of $\Gamma$, (c) for each $r, 0<r<1$, the set on which $\mu_{n}$ vanishes lies outside of $\overline{\Delta(0 ; r)}$ and $\mu_{n}$ is continuous at each point of $\overline{\Delta(0 ; r)}$ for $n$ sufficiently large. Of course, we put aside the trivial case where $E=\varnothing$. The existence of $\left(\mu_{n}\right)$ may be established as follows. We first introduce a locally finite covering of the fundamental domain $D$ of $\Gamma$ [here the set of $z$ in $\Delta(0 ; 1)$ whose non-euclidean distance to 0 does not exceed its distance to $\tau 0, \tau \in \Gamma$-it will be assumed that $\Gamma$ does not possess a member distinct from the identity having 0 as a fixed point] by noneuclidean disks $\delta$ with centers in $D$ satisfying: (1) only a finite number of the $\delta$ have a non-euclidean radius exceeding a given positive number, $(2)$ the closure of each $\delta$ lies in a non-euclidean disk with the same center in which two points are equivalent only if they differ by a non-euclidean rotation of $\Gamma$ having the given center as fixed point. For each $\delta$ such that $E \cap\left(U_{\tau \in \Gamma} \tau \delta\right) \neq \varnothing$ we construct as above an $S$ - $K$ metric invariant with respect to the transformations of $I$, the set on which it vanishes being $E \cap\left(U_{\tau \in \Gamma} \tau \delta\right)$. [We assume that there are infinitely many such $\delta$. The finite case may be treated in the same manner as the case where the fundamental domain of $\Gamma$ is compact.] We take $\left(\mu_{n}\right)_{1}^{\infty}$ as a univalent enumeration of the so constructed metrics. It has the stated properties. The convention of $\S \mathbf{1 4}$ concerning conformal metrics on $\Delta(0 ; 1)$ will be understood to prevail.

For each positive integer $k$ let $n(k)$ denote the smallest $n$ such that for $m \geq n$ the closure of the set where $\mu_{m}$ vanishes does not intersect $\overline{\Delta\left(0 ; 1-2^{-k}\right)}$, 
and $\mu_{m}$ is continuous on $\overline{\Delta\left(0 ; 1-2^{-k}\right)}$. We note that $(n(k))_{1}^{\infty}$ is non-decreasing and that $\lim _{k \rightarrow \infty} n(k)=\infty$. For each $k$ satisfying $n(k)<n(k+1)$ we choose a positive number $p_{k}<2^{-k}$ such that

$$
\left|\sum_{n(k) \leq n<n(k+1)} \log \mu_{n}(z)\right|<\frac{n(k+1)-n(k)}{p_{k} 2^{k}}, \quad|z| \leq 1-2^{-k} .
$$

Let $l$ be such that $n(l+1)>n(l)>1$. We introduce

$$
\lambda=\left(\prod_{1 \leq n<n(l)} \mu_{n}\right)^{\frac{1-\Sigma p_{k}}{n(l)-1}} \prod_{k}\left[\left(\prod_{n(k) \leq n<n(k+1)} \mu_{n}\right)^{\frac{p_{k}}{n(k+1)-n(k)}}\right]
$$

where the $k$ are taken as the positive integers satisfying: $k \geq l, n(k+1)>n(k)$. It is readily verified that thanks to (15.1), the right-hand side of (15.2) is convergent for $|z|<1$ and defines an upper semi-continuous function whose zero set is $E$ and which yields a conformal metric invariant under the transformations of $\Gamma$. There remains to be considered the mean-value property. To that end, we consider a point $a$ for which $\lambda(a) \neq 0$ and set $v(z)=\log \lambda(a+z)$. For $r>0$ sufficiently small we have

$$
\begin{aligned}
m(r ; v)-v(0) & \geq r^{2} \sum_{1}^{n(l)-1}\left[\frac{1-\sum p_{k}}{n(l)-1}\right]\left[\mu_{n}(a)\right]^{2} \\
& +r^{2} \sum_{k}\left[\frac{p_{k}}{n(k+1)-n(k)}\right]\left\{\sum_{n(k) \leq n<n(k+1)}\left[\mu_{n}(a)\right]^{2}\right\}
\end{aligned}
$$

On applying the weighted form of the theorem of arithmetic and geometric means, we see that for $r$ sufficiently small

$$
m(r ; v)-v(0) \geq r^{2}[\lambda(a)]^{2} .
$$

We conclude that $\lambda$ defines an $S-K$ metric.

It is now easy to see that if a $G_{\delta}$ of zero capacity, say $X$, is given on a Riemann surface $F$ that admits a hyperbolic metric, then there exists an $S \cdot K$ metric on $F$ which vanishes precisely on $X$. It suffices to introduce a conformal universal covering $\varphi$ of $F$ with domain $\Delta(0 ; 1)$ and to note that $\varphi^{-1}(X)$ is a $G_{\delta}$ of zero capacity invariant under the conformal automorphisms of $\Delta(0 ; 1)$ which leave $\varphi$ fixed. The argument is completed by applying the construction of the present section and $\S 8(\mathrm{e})$.

16. Boundary problems. The Perron method may be employed to treat 
boundary problems for $C^{\prime \prime}$ conformal metrics of constant curvature -4 . We consider the following situation. Let $\Omega$ denote a region contained in a compact Riemann surface $F$. The frontier of $\Omega$ is to consist of a finite number $(\geq 1)$ of mutually disjoint Jordan curves $\gamma_{1}, \ldots, \gamma_{n}$. For the sake of simplicity we suppose that $\cup \gamma_{k}=\operatorname{fr}(F-\Omega)$ although we may easily treat by the present methods boundary problems in the case of two-sided approach to a given boundary component. Let $\beta$ denote a continuous non-vanishing conformal metric on fr $\Omega$. We ask: Does there exist a continuous non-vanishing conformal metric $\lambda$ on $\Omega$ satisfying: (a) its restriction to $\operatorname{fr} \Omega$ is $\beta$, (b) $\lambda^{\Omega}$ is $C^{\prime \prime}$ and of constant curvature -4 . Of course, Theorem 2.1 assures us that there is at most one such conformal metric. We turn to the existence problem.

To that end, let $A_{k}$ denote a Jordan annulus, $\gamma_{k} \subset A_{k} \subset F$, which has the property that $\gamma_{k}$ separates in $\bar{A}_{k}$ the components of $\operatorname{fr} A_{k}, k=1, \ldots, n$. We suppose as we may that the $\bar{A}_{k}$ are mutually disjoint. Let $\tau_{k}$ denote a univalent conformal map of $A_{k}$ onto a plane annulus. The boundary components of $\tau_{k}\left(A_{k}\right)$ are separated by $\tau_{k}\left(\gamma_{k}\right)$. Let $\beta_{k}$ denote the continuous function induced on $\tau_{k}\left(\gamma_{k}\right)$ from $\beta$ by $\tau_{k}^{-1}$. There exists a Jordan annulus $B_{k} \subset \tau_{k}\left(A_{k}\right)$ whose frontier contains $\tau_{k}\left(\gamma_{k}\right)$ such that $\tau_{k}^{-1}\left(B_{k}\right) \subset \Omega$ and the boundary value problem with respect to $B_{k}$ for $\Delta u=4 e^{2 u}$ and continuous boundary function with maximum $\leq \max \log \beta_{k}$ admits a solution.

In this connection we note that we may choose $B_{k}$ in such a manner that

$$
\max _{z \in B k} \iint_{B k} g(z, \zeta) d S_{\zeta}
$$

does not exceed a given positive number, g being Green's function for $B_{k}$. Such a choice of $B_{k}$ may and will be made by considering first the harmonic measure $\boldsymbol{u}_{k}$ of $\tau_{k}\left(\gamma_{k}\right)$ with respect to $\tau_{k}\left(A_{k} \cap \Omega\right)$ and taking $B_{k}$ as $\left\{1>\boldsymbol{u}_{k}>1-\eta\right\}$ where $\eta, 0<\eta<1$. is taken so small that $(16.1)$ is fulfilled. The reasoning of $\S 4$ is now applicable. It is to be observed that the integral in (16.1) vanishes continuously on $\operatorname{fr} B_{k}$.

To continue, we introduce a $C^{\prime \prime}$ conformal metric $\mu$ of constant curvature -4 on a region containing $\bar{\Omega}$ and choose a positive number $c \leq 1$ so that the values induced from $c \mu$ by $\tau_{k}^{-1}$ on $\tau_{k}(\gamma)$ are less than $\min \beta_{k}, k=1, \ldots, n$. There exists a continuous conformal metric $\sigma_{k}$ on $\overline{\tau_{k}^{-1}\left(B_{k}\right)}$ satisfying: (a) the restriction of $\sigma_{k}$ to $\tau_{k}^{-1}\left(B_{k}\right)$ is $C^{\prime \prime}$ and of constant curvature -4 , (b) the restric- 
tion of $\sigma_{k}$ to $\gamma_{k}$ is equal to the restriction of $\beta$ to $\gamma_{k}$, (c) the restriction of $\sigma_{k}$ to $\gamma_{k}^{\prime}$, the other component of $\operatorname{fr}_{k}^{-1}\left(B_{k}\right)$, is less than the restriction of $c \mu$ to $r_{k}^{\prime}$. Consequently, there exists a continuous conformal metric on $\Omega$ whose restriction to $\operatorname{fr} \Omega$ is $\beta$ and whose restriction to $\Omega$ is $S-K$. Such a conformal metric is the conformal metric $\nu$ on $\Omega$ whose restriction to $\Omega-U \overline{\tau_{k}^{-1}\left(B_{k}\right)}$ is the restriction of $c \mu$ to this set and whose restriction to $\overline{\tau \bar{k}^{-1}\left(B_{k}\right)}$ is the maximum of $\sigma_{k}$ and the restriction of $c \mu$ to $\overline{\tau-1}\left(B_{k}\right), k=1, \ldots, n$.

Let $\alpha$ denote the continuous non-vanishing conformal metric on $\Omega$ whose restriction to $\operatorname{fr} \Omega$ is $\beta$ and whose restriction to $\Omega$ is euclidean. The uniqueness of $\alpha$ follows from the maximum principle for harmonic functions. To establish the existence, we introduce an analytic differential $\omega$ on $\Omega$ which does not vanish and note that there exists a continuous function $h$ on $\dot{\Omega}$, harmonic in $\Omega$ and reducing to $\log (\beta /|\omega|)$ on fr $\Omega$. The obvious notational gloss is to be made. We have: $\alpha=(\exp \circ h)|\omega|$.

We note that a majorant principle prevails for $S-K$ metrics and non-vanishing euclidean metrics dominating them on the boundary - the domination extending to the interior.

Now let $\lambda$ denote the upper envelope of the Perron family generated by the restriction of $\nu$ to $\Omega$. The desired existence result follows from

$$
\nu^{\Omega} \leq \lambda<\alpha^{\Omega} .
$$

17. Convexity properties. In the theory of subharmonic functions the convexity properties of the maximum or mean on a circumference are of interest. Corresponding properties in the case of $S-K$ metrics yield useful information, especially in the study of the local behavior of an $S-K$ metric near an isolated singularity. Even sharper information may be obtained in the case of a $C^{\prime \prime}$ conformal metric of constant curvature - 4. As far as I am aware, such results have not hitherto been explicitly stated. They complement in one respect the formulation of the Schwarz-Picard problem [cf. Ch. 2, §19], for it is not at all evident from the work of Picard to what extent the nature of the possible isolated singularities of $C^{\prime \prime}$ conformal metrics of constant negative curvature was taken into account by him.

Let us start with the elementary non-linear differential equation

$$
y^{\prime \prime}=4 e^{2 y}
$$


-its significance for our fundamental equation $\Delta u=4 e^{2 u}$ is obvious-and note certain basic properties of the solutions. We fix a point $(a, b)$ in the plane, and for $\alpha$ real we let $y_{\alpha}$ denote the solution of (17.1) whose domain is a semiclosed interval with lefthand endpoint $a$ [possibly $\{a \leq x\}$ ], satisfying $y(a)=b$, $y^{\prime}(a)=\alpha$, and having a maximal domain of the stated type. Given $\xi>a, \eta$ arbitrary real, there exists a unique $\alpha$ such that

$$
y_{\alpha}(\xi)=\eta \text {. }
$$

If $\alpha>-2 e^{b}$, the domain of $y_{\alpha}$ is bounded. Its right hand endpoint may even be determined by quadratures. If $\alpha \leq-2 e^{b}$, the domain is unbounded. If $\alpha=-2 e^{b}$,

$$
y_{\alpha}(x)=\log \frac{1}{x}+c+o(1), \quad x \text { large, }
$$

while if $\alpha<-2 e^{b}$,

$$
y_{\alpha}(x)=-x \sqrt{\alpha^{2}-4 e^{2} b}+o(x), \quad x \text { large. }
$$

These are only preliminary observations. For our local studies we shall be concerned rather with

$$
Y^{\prime \prime}+\frac{1}{r} Y^{\prime}=4 e^{2 Y}
$$

and shall consider solutions with domain of the form $\{10 \leq) B<r \leq A\}, A=e^{-a}$, satisfying $Y(A)=b+a$, the domain being maximal. The mapping, $Y \rightarrow y$, specified by

$$
y(x)=Y\left(e^{-x}\right)-x, \quad a \leq x<-\log B,
$$

maps the set of solutions of (17.5) taken into account onto the set of the $y_{\alpha}$. The antecedent, $Y_{\alpha}$, of $y_{\alpha}$ satisfies $Y_{\alpha}^{\prime}(A)=-(\alpha+1) / A$. If $\alpha>-2 e^{b}$, the lefthand endpoint of the domain of $Y_{\alpha}$ is positive. Otherwise it is zero. From (17.3) and (17.4), we conclude

$$
Y_{\alpha}(r)=\log \frac{1}{r}-\log \left(\log \frac{1}{r}\right)+c+o(1), \alpha=-2 e^{b}
$$

and

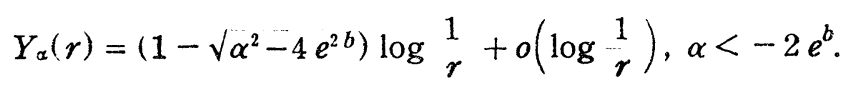

Let $f$ denote a real-valued function whose domain is of the form $\{0<r \leq A\}$. 
We say that $f$ is convex relative to the family of solutions of (17.5), or more simply, that $f$ is *-convex provided that whenever $0<B<C \leq A$ and $Y$ is the solution of (17.5) with domain $\{B \leq r \leq C\}$ which satisfies

$$
Y(B)=f(B), \quad Y(C)=f(C),
$$

then

$$
f(r) \leq Y(r), \quad B \leq r \leq C .
$$

[We define $*$-convexity analogously for a function with domain $\{0<r<A\}$.] We now introduce for each $B, 0<B<A$ the unique solution of (17.5) with domain $\{B \leq r \leq A\}$ which satisfies

$$
Y(B)=f(B), \quad Y(A)=f(A),
$$

and denote it by $Z_{B}$. We note that for $B<r<A, B \rightarrow Z_{B}(r)$ is non-increasing. Letting $B \rightarrow 0$, we conclude that $f$ is dominated by a solution of (17.5) on $\{0<r \leq A\}$ which agrees with $f$ at $A$. We let $\alpha_{0}$ be the least value of $\alpha$ for which $Y_{\alpha} \geq f$. We have $\alpha_{0} \leq-2 e^{f_{(A)}}$. Let $\beta=1-\left[\alpha_{0}^{2}-4 e^{2 f(A)}\right]^{1 / 2}$. We assert that

$$
\lim _{r \rightarrow 0} \frac{f(r)}{\log \frac{1}{r}}=\beta .
$$

To see this, we note that from $Y_{\alpha_{0}} \geq f$ and (17.7), (17.8) we have

$$
\limsup _{r \rightarrow 0} \frac{f(r)}{\log \frac{1}{r}} \leq \beta .
$$

On the other hand, if

$$
\liminf _{r \rightarrow 0} \frac{f(r)}{\log \frac{1}{r}}<\beta
$$

on taking $\alpha<\alpha_{0}$ satisfying

$$
1-\sqrt{\alpha^{2}-4 e^{2 f(A)}}>\liminf _{r \rightarrow 0} \begin{array}{r}
f(r), \\
\log \frac{1}{r}
\end{array}
$$

we see that $f \leq Y_{\alpha}$ and the minimal character of $\alpha_{0}$ is violated. We conclude (17.9).

Suppose now that $u$ is a $C^{\prime \prime}$ solution of $\Delta u=4 e^{2 u}$ on $\Delta(0 ; R)-\{0\}$. We propose to study 


$$
M(r)=\max u\left(r e^{i \theta}\right)
$$

and

$$
m(r)=\frac{1}{2 \pi} \int_{0}^{2 \pi} u\left(r e^{i \theta}\right) d \theta, \quad 0<r<R
$$

If $0<r_{1}<r_{2}<R$, the solution of (17.5) on $\left\{r_{1} \leq r \leq r_{2}\right\}$ taking the value $M\left(r_{k}\right)$ at $r_{k}(k=1,2)$ dominates $M(r)$ on $\left\{r_{1} \leq r \leq r_{2}\right\}$. Hence $r \rightarrow M(r)$ is *-convex on $\{0<r<R\}$. To treat the mean, we note that $m(r)$ satisfies

$$
m^{\prime \prime}+\frac{1}{r} m^{\prime}=\frac{4}{2 \pi} \int_{0}^{2 \pi} \exp \left[2 u\left(r e^{i \theta}\right)\right] d \theta \geq 4 e^{2 m},
$$

an inequality that implies the $*$-convexity of $m(r)$ on $\{0<r<R\}$ [11].

Suppose now that $\lambda$ is an $S-K$ metric on $F-\{a\}$. Let $\varphi$ denote a univalent conformal map of $\Delta(0 ; R)-\{0\}$ into $F-\{a\}$ which satisfies $\lim _{z \rightarrow 0} \varphi(z)=a$ and let $u=\log \lambda_{\varphi}$. Then, if $\lambda$ does not vanish identically, $M(r)$ and $m(r)$ are *-convex. For $M(r)$ the proof is the same as that indicated in the preceding paragraph. For $m(r)$ we note that if $0<r_{1}<r_{2}<R$, and $v$ is continuous on $\left\{r_{1} \leq|z| \leq r_{2}\right\}$, is $C^{\prime \prime}$ and satisfies $\Delta v=4 e^{2 v}$ in $\left\{r_{1}<|z|<r_{2}\right\}$, and dominates $u$ on $\boldsymbol{C}\left(0 ; r_{k}\right), k=1,2$, then $v$ dominates $u$ throughout $\left\{r_{1} \leq|z| \leq r_{2}\right\}$. Hence $\boldsymbol{m}(\boldsymbol{r})$ is dominated on $\left\{r_{1} \leq r \leq r_{2}\right\}$ by the solution of (17.5) which reduces at $r_{k}$ to the mean of $v$ on $C\left(0 ; r_{k}\right), k=1,2$. Now by the existence theorem for the boundary problem for $C^{\prime \prime}$ conformal metrics of constant curvature -4 there exists $v$ continuous on $\left\{r_{1} \leq|z| \leq r_{2}\right\}, C^{\prime \prime}$ and satisfying $\Delta v=4 e^{2 v}$ in $\left\{r_{1}<|z|<r_{2}\right\}$, and reducing to a given continuous function on $C\left(0 ; r_{k}\right), k=1,2$. Thanks to the upper semi-continuity of $u$, there exists a sequence of such $v$, say $\left(v_{n}\right)_{1}^{\infty}$, which is monotone decreasing and tends pointwise to $u$ on $C\left(0 ; r_{k}\right), k=1,2$. These remarks taken together imply that $m(r)$ is dominated on $\left\{r_{1} \leq r \leq r_{2}\right\}$ by the solution of (17.5) which reduces at $r_{k}$ to $m\left(r_{k}\right), k=1,2$.

It is to be observed that there is a marked contrast between the behavior near zero of $u$ and that of an unrestricted subharmonic function in $\Delta(0 ; R)-\{0\}$. In fact (17.9) yields for our present $u$

$$
\lim _{r \rightarrow 0} \frac{M(r)}{\log \frac{1}{r}} \leq 1,
$$

a restriction to which not all subharmonic functions on $\Delta(0 ; R)-\{0\}$ are subject. 
18. Isolatcd singularities of solutions of $\Delta u=4 e^{2 u}$. Let $u$ denote a continuous function on $\overline{\Delta(0 ; 1)}-\{0\}$ which is a $C^{\prime \prime}$ solution of $\Delta u=4 e^{2 u}$ in $\Delta(0 ; 1)-\{0\}$. We propose to study the behavior of $u$ near 0 .

Case 1. $\lim _{r \rightarrow 0} M(r) / \log \left(r^{-1}\right)<1$. Let $\alpha$ denote $\lim _{r \rightarrow 0} m(r) / \log \left(r^{-1}\right)$ and observe that $u$ admits a representation of the form

$$
u(z)=h(z)+\alpha \log \frac{1}{|z|}-T\left[4 e^{2 u}\right], \quad 0<|z|<1,
$$

(cf. $\S 4, r=1$ ), where $h$ is harmonic on $\Delta(0 ; 1)$. Thanks to the fact that

$$
u(z)<\alpha \log \frac{1}{|z|}+c
$$

for $z$ small, we conclude that $T\left[4 e^{2 u}\right]$ is continuous at the origin. Hence we have

$$
u(z)=\alpha \log \frac{1}{|z|}+c_{1}+o(1), \quad z \text { small, }
$$

a consequence of which is:

$$
\alpha=\lim _{r \rightarrow 0} \frac{M(r)}{\log \frac{1}{r}} .
$$

Note (18.2) refines (17.8)

It is easily seen that if $v$ satisfies the same conditions as $u$ and in addition $v \leq u$ and $\lim _{0}(v-u)=0$, then $v=u$. It suffices to employ the argument of Lemma 7.1 with obvious modifications.

Case 2. $\lim _{r \rightarrow 0} M(r) / \log \left(r^{-1}\right)=1$. Here the treatment is somewhat more delicate. At all events, the developments of Case 1 show that $\lim _{r \rightarrow 0} m(r) / \log \left(r^{-1}\right)$ $=1$.

We observe that there is a unique $u$ that takes a given constant value $c$ on $C(0 ; 1)$ and satisfies $\lim _{r \rightarrow 0} m(r) / \log \left(r^{-1}\right)=1$. The existence follows from the remarks concerning the solutions of (17.5). If $v$ is a second such function, we have

$$
v-u=T\left[4\left(e^{2 u}-e^{2 v}\right)\right],
$$

so that

$$
(v-u)^{+} \leq T\left[4 e^{2 u}\right] .
$$


On the other hand, $(v-u)^{+}$is subharmonic in $\Delta(0 ; 1)-\{0\}$ and $T\left[4 e^{2 u}\right]$ is a Green's potential in $\Delta(0 ; 1)$. Hence $(v-u)^{+}=0$ and by symmetry $u=v$.

We return to an unrestricted $u$ of Case 2 and introduce $a=\min u\left(e^{i \vartheta}\right)$ and for each $r, 0<r<1$, we introduce $v_{r}$ [resp. $V_{r}$, the continuous function on $\{r \leq|z| \leq 1\}$ reducing to $a$ [resp. $M(1)]$ on $C(0 ; 1)$ and to $u$ on $C(0 ; r)$ which further is $C^{\prime \prime}$ and satisfies $\Delta w=4 e^{2 w}$ in $\{r<|z|<1\}$. Let $v(z)=\lim _{r \rightarrow 0} v_{r}(z)$ and $V(z)=\lim _{r \rightarrow 0} V_{r}(z), 0<|z|<1$. We have

$$
v \leq u \leq V
$$

Further $v$ and $V$ are both of the form

$$
\log \frac{1}{|z|}-\log \left(\log \frac{1}{|z|}\right)+c+o(1)
$$

since each is a function of the type considered in the previous paragraph. We next observe that $u-v$ and $V-u$ are subharmonic and bounded on $\Delta(0 ; 1)-\{0\}$ and consequently admit subharmonic extension to $\Delta(0 ; 1)$. Since $V-v$ $=(V-u)+(u-v)$ admits continuous subharmonic extension to $\Delta(0 ; 1)$, it follows that $u-v$ and $V-u$ both possess a limit at 0 . Hence $u$ admits a representation of the form (18.4).

Let $\lambda$ be a $C^{\prime \prime}$ conformal metric of constant curvature -4 , on $F-E$ where $E$ is a discrete subset of $F$. Let $a \in E$. Let $\Omega$ denote a plane region for which 0 is an isolated frontier point. Let $\varphi$ denote a univalent conformal map of $\Omega$ into $F-E$ satisfying $\lim _{0} \varphi=a$. Then $\log \lambda_{\beta}$ admits a representation of the form (18.2) or (18.4) and the coefficient of $\log \left(|z|^{-1}\right)$ is independent of $\varphi$. We term the common value of the coefficient of $\log \left(|z|^{-1}\right)$ the index of $\lambda$ at $a$ and denote it by $\nu(a ; \lambda)$.

[The index of a euclidean metric $\lambda$ at a point $a$ is analogously defined as the coefficient of $\log \left(|z|^{-1}\right)$ in the expansion of $\log \lambda_{p}$ about 0 and will be denoted by $\nu(a ; \lambda)$ as above. It is to be observed that for euclidean metrics the index may assume arbitrary real values. The index of a euclidean metric will be employed only in the case where $\log \lambda_{p}$ is of the form $\alpha \log \left(|z|^{-1}\right)+h(z)$, $h$ harmonic at 0 .]

It is now easy to see that if $F-\{a\}$ possesses a hyperbolic metric $\gamma$, then $\nu(a ; \gamma)=1$. The property is well-known. For plane regions cf. [10]. Suppose that $\nu(a ; \gamma)<1$. Then we may modify $\gamma$ to obtain an $S \cdot K$ metric $\mu$ for which 
$\| l \gamma$ takes a value greater than one somewhere. In fact, if $\varphi$ is a univalent conformal map of $\Delta(0 ; 2)-\{0\}$ into $F-\{a\}$ satisfying $\lim _{n} \varphi=a$, it suffices to introduce a $C^{\prime \prime}$ solution $v$ of $\Delta v=4 e^{2 v}$ in $\Delta(0 ; 1)-\{0\}$ taking constant boundary values less than $\min _{C(0 ; 1)} \log \gamma_{\rho}$ on $C(0 ; 1)$ and of the form (18.4). We let $\mu$ denote the metric obtained when $\log \gamma_{p}(z)$ is replaced by $\max \left\{\log \gamma_{p}(z), v(z)\right\}$ for $0<|z|<1$. However it is not possible for such a metric $\mu$ to exist. Hence $\nu(a ; \gamma)=1$.

This result may be viewed as a corollary of the following theorem.

TheOREM 18.1: Let $F$ denote a non compact Riemann surface admitting a hyperbolic metric $\gamma$, and let $\Omega$ denote a component of the complement of a compact subset $K \subset F$, let $\gamma_{1}$ denote the hyperbolic metric of $\Omega$. Then $\gamma^{2} / \gamma_{1}$ has a positive lower bound in $\Omega-V$, where $V$ is a neighborhood of $K$.

\section{Chapter II. The problem of Schwarz-Picard}

19. The problem proposed by H. A. Schwarz and treated by Picard [13] may be stated in the language of the present paper as follows:

Given a compact Riemann surface $F, n$ distinct points $a_{1}, \ldots, a_{n}$ on $F$ and real numbers $\nu_{k} \leq 1, k=1, \ldots, n$. Does there exist a $C^{\prime \prime}$ conformal metric $\lambda$ of constant curvature -4 on $F-\left\{a_{1}, \ldots, a_{n}\right\}$ which satisfies

$$
\nu\left(a_{k} ; \lambda\right)=\nu_{k}, \quad k=1, \ldots, n \text { ? }
$$

To be exact, Picard envisages an equivalent problem for squares of metrics. Further, he treats the problem corresponding to that formulated here when $\nu_{k}<1$. It is also to be remarked that a local representation of the form (18.2) is assumed outright by Picard as an expression of the local behavior of the metric in the neighborhood of a singularity.

An elementary construction of a $C^{\prime \prime}$ conformal metric in the plane with singularities of the type considered by Picard and of curvature $\leq-1$ at the non-singular points was given by R. M. Robinson [15].

We now give an account of the above formulated Schwarz-Picard -problem in terms of the methods and ideas of the present paper.

20. Uniqueness. The unicity question may be handled very rapidly. Suppose that $\lambda$ and $\mu$ satisfy the specified conditions. Then $\lambda / \mu$ admits a continuous 
non-vanishing extension to $F$ and $\log (\lambda / \mu)$ is subharmonic and consequently constant since $F$ is compact. The constant value is not positive, for otherwise the curvature would not be -4 for both $\lambda$ and $\mu$. Hence $\lambda \leq \mu$. By symmetry $\lambda=\mu$.

21. The $\lambda$-area of $F-\left\{a_{1}, \ldots, a_{n}\right\}$. It is classical that the area of $F-\left\{a_{1}, \ldots, a_{n}\right\}$ in the sense of a metric $\lambda$ meeting the conditions of the problem is simply

$$
\frac{\pi}{2}\left[\chi(F)+\sum_{k=1}^{n} \nu\left(a_{k} ; \lambda\right)\right],
$$

where $\chi(F)$ is the Euler characteristic of $F(=2 g-2, g$ being the genus of $F)$. Hence since the $\lambda$-area is positive, we obtain

Theorem 21.1: A necessary condition for the problem of Schwarz-Picard to possess an affirmative answer is that

$$
\sum_{k=1}^{n} \nu_{k}>-\chi(F)
$$

22. The condition (21.2) is sufficient for the problem of Schwarz-Picard to have an affirmative answer. This aspect of the solution of the problem is, as might be expected, more difficult. We first establish several lemmas. In these lemmas we understand that $n \geq 1$.

LEMMA 22.1: Given $\left(\nu_{1}^{0}, \ldots, \nu_{n}^{0}\right)$ such that there exists a $C^{\prime \prime}$ conformal metric $\lambda$ of constant curvature -4 on $F-\left\{a_{1}, \ldots, a_{n}\right\}$ satisfying $\nu\left(a_{k} ; \lambda\right)=\nu_{k}^{0}$, $k=1, \ldots, n$. Then for each $n$-tuple $\left(\nu_{1}, \ldots, \nu_{n}\right)$ satisfying $\nu_{k}^{\circ} \leq \nu_{k} \leq 1, k=1$, $\ldots, n$, there exists a $C^{\prime \prime}$ conformal metric $\mu$ of constant curvature -4 on $F-\left\{a_{1}, \ldots, a_{n}\right\}$ satisfying $\nu\left(a_{k} ; \mu\right)=\nu, k=1, \ldots, n$.

Proof: We modify $\lambda$ locally near $a_{k}$, if $\nu_{k}>\nu_{k}^{0}$, to obtain an $S-K$ metric $\lambda^{*}$ on $F-\left\{a_{1}, \ldots, a_{n}\right\}$ whose restriction to a deleted neighborhood of $a_{k}$ is $C^{\prime \prime}$, of constant curvature -4 , and such that $\nu\left(a_{k} ; \lambda^{*}\right)=\nu_{k}$. Such a modification is easily constructed on noting that there exists a $C^{\prime \prime}$ solution of $\Delta u=4 e^{2 u}$ on $\Delta(0 ; 1)-\{0\}$ which takes an assigned constant boundary value on $C(0 ; 1)$, and is of the form (18.2) with $\nu_{k}=\alpha$ if $\nu_{k}<1$, and is of the form (18.4) if $\nu_{k}=1$. [cf. last paragraph of $\$ 18$.]

Now let $\varphi_{k}$ denote a univalent conformal map of $\Delta(0 ; 2)$ into $F, \varphi_{k}(0)=a_{k}$, 
the $\varphi_{k}[\Delta(0 ; 2)]$ being mutually disjoint. Let $\mu_{k}$ denote the maximal $C^{\prime \prime}$ conformal metric on $\varphi_{k}[\Delta(0 ; 1)-\{0\}]$ of constant curvature -4 satisfying $\nu\left(a_{k} ; \mu_{k}\right)$ $=\nu_{k}$. The existence of $\mu_{k}$ is readily established with the aid of a suitable solution of (17.5), i.e. $Y$ on $\{0<r<1\}$ satisfying $\lim _{r \rightarrow 1} Y(r)=+\infty, Y(r)=\nu_{k} \log (1 / r)$ $+c+o(1)$ if $\nu_{k}<1$, and otherwise $Y(r)=\log (1 / r)-\log [\log (1 / r)]+c+o(1)$, $r$ small.

The Perron family generated by $\lambda^{*}$ has the property that $\mu_{k}$ dominates the restriction to $\varphi_{k}[\Delta(0 ; 1)-\{0\}]$ of each member of the family. The upper envelope of the family is the desired $\mu$.

LemMa 22.2: Under the hypotheses of Lemma 22.1 there exists $a C^{\prime \prime}$ conformal metric $\mu$ of constant curvature -4 on $F-\left\{a_{1}, \ldots, a_{n}\right\}$ satisfying

$$
\nu\left(a_{k} ; \mu\right)<\nu_{k}^{0}, \quad k=1, \ldots, n .
$$

Proof: Let $\delta$ satisfy $\sum\left(\nu_{k}^{0}-\delta\right)=-\chi(F)$, so that $\delta>0$. From standard properties of abelian differentials on $F$ and the fact that there exists a harmonic function $h$ on $F$ less two given distinct points at which $h$ has logarithmic singularities it follows that there exists a non-vanishing euclidean metric $\omega$ on $F-\left\{a_{1}, \ldots, a_{n}\right\}$ which satisfies $\nu\left(a_{k} ; \omega\right)=\nu_{k}^{0}-\delta, k=1, \ldots, n$. Using property (e) $\S 10$ we infer the existence of an $S-K$ metric $\lambda^{*}$ on $F-\left\{a_{1}, \ldots, a_{n}\right\}$ which satisfies for small $z$ the condition

$$
\log \lambda_{? k}^{*}(z)=\alpha_{k} \log \frac{1}{|z|}-\beta_{k} \log \left(\log \frac{1}{|z|}\right)+\gamma_{k}+o(1) .
$$

Here $\varphi_{k}$ is a univalent conformal map of $\Delta(0 ; 2)-\{0\}$ into $F-\left\{a_{1}, \ldots, a_{n}\right\}$ satisfying $\lim _{0} \varphi_{k}(z)=a_{k}$ and $\alpha_{k}<\nu_{k}^{0}$. By a paraphrase of the argument of Lemma 22.1 we see that the upper envelope of the Perron family generated by $\lambda^{*}$ is an admitted $\mu$. It suffices to replace $\mu_{k}$ of that lemma by a conformal metric meeting all the same conditions save that its index at $a_{k}$ is to be $\alpha_{k}$.

Lemma 22.3: Let $\left(\alpha_{1}, \ldots, \alpha_{n}\right)$ denote an $n$-tuple of real numbers satisfying $\sum \alpha_{k}=-\chi(F)$ and $\alpha_{k}<1, k=1, \ldots, n$. Then for each $n$-tuple $\left(\nu_{1}, \ldots, \nu_{n}\right)$ satisfying

$$
\alpha_{k}<\nu_{k} \leq 1, \quad k=1, \ldots, n,
$$

there exists a $C^{\prime \prime}$ conformal metric $\lambda$ of constant curvature -4 on $F-\left\{a_{1}, \ldots\right.$, $a_{n}$ ) satisfying 


$$
\nu\left(a_{k} ; \lambda\right)=\nu_{k} .
$$

This is the essential lemma for the sufficiency theorem.

Proof: Let $E$ denote the set of $n$-tuples $\left(\nu_{1}, \ldots, \nu_{n}\right)$ satisfying (22.2). Clearly $E$ is convex. A fortiori $E$ is connected. We employ an argument based on the connectedness of $E$. Let $A$ denote the set of $n$-tuples $\left(\nu_{1}, \ldots, \nu_{n}\right) \in E$ for which there exists $\lambda$ meeting the specified conditions. Now $(1, \ldots, 1) \in A$. [If $\chi(F)=-2, n \geq 3$ ]. Further Lemmas 22.1 and 22.2 show that $A$ is open in the sense of the relative topology of $E$.

It remains to show that $A$ is closed in the sense of the relative topology of $E$. To that end, we consider a sequence $\left(\left(\nu_{1}^{j}, \ldots, \nu_{n}^{j}\right)\right)_{1}^{\infty}$ of members of $A$ tending to $\left(\nu_{1}^{0}, \ldots, \nu_{n}^{0}\right) \in E$ and show that $\left(\nu_{1}^{0}, \ldots, \nu_{n}^{0}\right) \in A$. It then follows that $A$ is closed in the sense of the relative topology of $E$. Let $\lambda^{j}$ denote the $C^{\prime \prime}$ metric of constant curvature -4 on $F-\left\{a_{1}, \ldots, a_{n}\right\}$ which satisfies $\nu\left(a_{k} ; \lambda^{j}\right)=\nu_{k}^{j}, k=1, \ldots, n$. By the normal family property of the $C^{\prime \prime}$ conformal metrics of constant curvature -4 there exists a subsequence of $\left(\lambda^{j}\right)$, which we may as well take to be $\left(\lambda^{j}\right)$ itself, converging uniformly in the sense defined earlier in $F-\left\{a_{1}, \ldots, a_{n}\right\}$ to either the identically zero metric or a $C^{\prime \prime}$ metric of constant curvature -4 . The $\lambda^{j}$-area of $F-\left\{a_{1}, \ldots, a_{n}\right\}$ namely

$$
\frac{\pi}{2}\left[\chi(F)+\sum_{k=1}^{n} \nu_{k}^{j}\right]
$$

has a positive lower bound independent of $j$. If $\left(\lambda^{j}\right)$ tended to the zero metric, the $\lambda^{j}$-area of $F-\left\{a_{1}, \ldots, a_{n}\right\}$ would tend to 0 as $j \rightarrow \infty$. In fact, let $\varphi_{k}$ denote a univalent conformal map of $\Delta(0 ; 2)$ into $F, \varphi_{k}(0)=a_{k}, k=1, \ldots, n$, the $\varphi_{k}[\Delta(0 ; 2)]$ being disjoint. Then given $r, 0<r<2$, for $j$ sufficiently large the $\lambda^{j}$-area of $F-\left\{a_{1}, \ldots, a_{n}\right\}$ would be less than twice the sum of the areas of the $\varphi_{k}[\Delta(0 ; r)]-\left\{a_{k}\right\}$ in the sense of the hyperbolic metric of $F-\left\{a_{1}, \ldots, a_{n}\right\}$. This is impossible for $r$ sufficiently small.

Hence $\left(\lambda^{j}\right)$ tends to a $C^{\prime \prime}$ conformal metric $\lambda$ of constant curvature -4 on $F-\left\{a_{1}, \ldots, a_{n}\right\}$. Using the $\varphi_{k}$ of the preceding paragraph, we put down conformal metrics $\mu_{k}^{\prime}$ and $\mu_{k}^{\prime \prime}$ on $\varphi_{k}[\overline{\Delta(0 ; 1)}]-\left\{a_{k}\right\}$ satisfying the following conditions: (a) each be continuous; (b) the restriction of each to $\varphi_{k}[\Delta(0 ; 1)]$ - $\left\{a_{k}\right\}$ be $C^{\prime \prime}$ of constant curvature -4 ; (c) $\nu_{\nu}\left(a_{k} ; \mu_{k}^{\prime}\right)<\nu_{k}^{0}<\nu\left(a_{k} ; \mu_{k}^{\prime \prime}\right)$ if $\nu_{k}^{0}<1$, and otherwise $\nu\left(a_{k} ; \mu_{k}^{\prime}\right)<\nu\left(a_{k} ; \mu_{k}^{\prime \prime}\right)=1 ;$ (d) $\mu_{k}^{\prime}<\lambda<\mu_{k}^{\prime \prime}$ on $\varphi_{k}[C(0 ; 1)]$, the 
obvious gloss concerning restrictions being made. It follows that for $j$ sufficiently large

$$
\mu_{k}^{\prime}<\lambda^{j}<\mu_{k}^{\prime \prime}
$$

on $\varphi_{k}\left[\Delta(0 ; 1]-\left\{a_{k}\right\}\right.$. Hence we have on the same set

$$
\mu_{k}^{\prime}<\lambda<\mu_{k}^{\prime \prime}
$$

We conclude that

$$
\nu\left(a_{k} ; \mu_{k}^{\prime}\right) \leq \nu\left(a_{k} ; \lambda\right) \leq \nu\left(a_{k} ; \mu_{k}^{\prime \prime}\right)
$$

and hence, that

$$
\nu\left(a_{k} ; \lambda\right)=\nu_{k}^{\circ}, \quad k=1, \ldots, n,
$$

using the arbitrariness of $\nu\left(a_{k} ; \mu_{k}^{\prime}\right)$ and $\nu\left(a_{k} ; \mu_{k}^{\prime \prime}\right)$. Lemma 22.3 follows.

The solution of the Schwarz-Picard problem is now readily completed. We put aside the case where $n=0$. It is cared for by the hyperbolic metric. Given $\left(\nu_{1}, \ldots, \nu_{n}\right), \nu_{k} \leq 1, k=1, \ldots, n$, satisfying $(21.2)$, we take $\left(\alpha_{1}, \ldots\right.$, $\left.\alpha_{n}\right)$. satisfying $\sum \alpha_{k}=-\chi(F)$ and $\alpha_{k}<\nu_{k}, k=1, \ldots, n$, and thereupon apply Lemma 22.3.

\section{Chapter III. Applications to Conformal Mapping}

23. From this point on our central interest will be the application of conformal metrics, generally $C^{\prime \prime}$ with constant curvature -4 , to the study of conformal maps of Riemann surfaces, and more particularly to conformal maps and meromorphic functions with domain $\Delta(0 ; 1)$.

24. Nehari's generalization of the lemma of Schwarz. We quote Nehari's theorem [9]:

"Let $w=f(z)$ be a non-uniform function, regular for $|z|<1$ apart from $a$ finite number of algebraic branch points and let $f^{\prime}(z)$ be finite everywhere in $|z|<1$; let further, for all determinations of $f(z),|f(z)| \leq 1$ for $|z|<1$. Then we have

$$
\left|f^{\prime}(0)\right| \leq 1
$$

for all the different values $f^{\prime}(0)$ may assume. The case $\left|f^{\prime}(0)\right|=1$ can only happen for $f(z) \equiv K z,|K|=1$."

Nehari's theorem will now be considered from the point of view of our present study. It will be seen to admit considerable generalization. The following 
reworking of Nehari's theorem indicates the nature of our approach:

Let $f$ denote an $n$-sheeted conformal map of a Riemann surface $F$ onto $\Delta(0 ; 1)$ and let $g$ denote an analytic function of modulus $<1$ on $F$ which satisfies the condition $n(p ; f) \leq n(p ; g), p \in F$. [Here " $n(p ; f)$ " denotes the multiplicity of $f$ at $p$.$] Let \gamma$ denote the hyperbolic metric of $\Delta(0 ; 1)$. Then $[\gamma, g] \leq[\gamma, f]$. If the two sides are equal, $g=T \circ f$ where $T$ is a conformal automorphism of $\Delta(0 ; 1)$.

We remark that $[\gamma, f]$ is the maximal conformal metric on $F$ whose restriction to $F$ less the points at which $f$ is ramified is $C^{\prime \prime}$ of constant curvature -4 and which has index $1-n(p ; f)$ at each point $p$ with $n(p ; f)>1$. This is the essential fact of the part of the theorem concerning the inequality. It suffices to consider for $r$ less than but sufficiently near one the metric induced from the hyperbolic metric of $\Delta(0 ; r)$ by the restriction of $f$ to $f^{-1}[\Delta(0 ; r)]$, only $r$ such that $f$ is not ramified over $C(0 ; r)$ being taken into account.

More generally, a theorem of this type is available when we have (as above) a maximal conformal metric induced from a hyperbolic metric. We have

Theorem 24.1: Let $f_{k}$ denote a conformal map of a Riemann surface $F$ into a Riemann surface $G_{k}$ possessing a hyperbolic metric $\gamma_{k}, k=1,2$. Suppose that $n\left(p ; f_{1}\right) \leq n\left(p ; f_{2}\right), p \in F$, and that $\left[\gamma_{1}, f_{1}\right]$ is the maximal conformal metric on $F$ whose restriction to $F-\left\{n\left(p ; f_{1}\right)>1\right\}$ is $C^{\prime \prime}$ of constant curvature -4 with index $1-n\left(p ; f_{1}\right)$ at $p$ satisfying $n\left(p ; f_{1}\right)>1$. Then

$$
\left[r_{2}, f_{2}\right] \leq\left[\gamma_{1}, f_{1}\right] \text {. }
$$

In addition, if $G_{1}$ and $G_{2}$ are conformally equivalent to $\Delta(0 ; 1)$ and equality holds in (24.1), $f_{2}=T \circ f_{1}$, where $T$ is a univalent conformal map of $G_{1}$ onto $G_{2}$.

We need only verify the last assertion. We assume, as we may, that $G_{1}=G_{2}=\Delta(0 ; 1)$. Let $p_{0} \in F$ be such that $n\left(p_{0} ; f_{1}\right)=1$ and let $T$ be analytic on a region containing $f_{1}\left(p_{0}\right)$, be of modulus less than one, and satisfy $T\left[f_{1}(p)\right]$ $=f_{2}(p), p$ near $p_{0}$. Then

$$
\frac{\left|T^{\prime}(z)\right|}{1-|T(z)|^{2}}=\frac{1}{1-|z|^{2}}
$$

for $z$ near $f_{1}\left(p_{0}\right)$. The equality (24.2) implies that $T$ is the restriction of a conformal automorphism of $\Delta(0 ; 1)$ and the assertion follows.

[For the sake of completeness we indicate a proof. Cf. [9]. We may assume 
that $f_{1}\left(p_{0}\right)=0$ and $T(0)=0$. The equality (24.1) then yields the result that the hyperbolic distance between 0 and $T(z)$ is equal to the hyperbolic distance between 0 and $z$ for $z$ small. Hence $|T(z)|=|z|$ for $z$ small and $T$ is the restriction of a conformal automorphism of $\Delta(0 ; 1)$. This argument will be used later [§29].]

We now see that interest centers on determining a comprehensive class of conformal maps that induce maximal metrics.

25. A class of conformal maps. An interior transformation $f$ of a surface $F$ into a surface $G$ will be said to be locally of island type (inselartig im kleinen) provided that for each $q \in G$ there exists a disk $\Delta, q \in \Delta$, such that each component of $f^{-1}(\Delta)$ is relatively compact. It is obvious that the class of maps that are locally of island type embraces the class of maps of constant finite valence as well as many frequently considered interior transformations (such as branched coverings).

We first prove

THEOREM 25.1: Let $f$ be a conformal map of a Riemann surface $F$ into a Riemann surface $G$ and suppose that $f$ is locally of island type. Then

(a) If $G$ possesses a hyperbolic metric $r,[r, f]$ is the maximal conformal metric on $F$ whose restriction to $F-\{n(p ; f)>1\}$ is $C^{\prime \prime}$ of constant curvature -4 and has index $1-n(p ; f)$ at $p$ satisfying $n(p ; f)>1$.

(b) If there does exist a $C^{\prime \prime}$ conformal metric of constant curvature -4 on $F-\{n(p ; f)>1\}$ with index $1-n(p ; f)$ at $p$ satisfying $n(p ; f)>1$, then $G$ possesses a hyperbolic metric.

Proof: Let us first treat (a). Let $\lambda$ denote the maximal conformal metric to which reference is made. We shall "induce" from $\lambda$ an $S-K$ metric $\mu$ on $G$ satisfying

$$
\lambda \leq[\mu, f] .
$$

Since $\mu \leq \gamma$ and $[\gamma, f] \leq \lambda$, we infer $[\gamma, f]=\lambda$.

The $S-K$ metric $\mu$ is introduced as follows. Given $q \in G$ we assign a uniformizer $\varphi_{q}, \varphi_{q}(0)=q$, with domain $\Delta(0 ; 1)$ such that each component $\Omega$ of $f^{-1}\left\{\varphi_{q}[\Delta(0 ; 1)]\right\}$ is relatively compact. We define

$$
M\left(z ; \varphi_{q}, \Omega\right)
$$


at a point $z \in \Delta(0 ; 1)$ for which $f_{\Omega}$, the restriction of $f$ to $\Omega$, is not ramified over $\varphi_{q}(z)$, to be the maximum of the values $\lambda_{\psi}(z)$ where $\psi$ is a uniformizer of $F$ whose domain contains $z$ and which satisfies $\varphi_{q}^{-1} \circ f_{\Omega} \circ \psi(w) \equiv w$. At the remaining points of $\Delta(0 ; 1), M\left(z ; \varphi_{q}, \Omega\right)$ is defined by taking limiting values. So defined $z \rightarrow M\left(z ; \varphi_{a}, \Omega\right)$ is the identity-scale [cf. $\S 1$ ] of an $S$ - $K$ metric on $\Delta(0 ; 1)$. Hence

$$
M\left(z ; \varphi_{q}, \Omega\right) \leq\left(1-|z|^{2}\right)^{-1}
$$

We introduce

$$
z \rightarrow \sup _{\Omega} M\left(z ; \varphi_{q}, \Omega\right), \quad|z|<1,
$$

and let $M_{\varphi_{y}}$ denote its upper limit function. There exists a conformal metric $\mu$ on $F$ satisfying $\mu_{q_{q}}=M_{\varphi_{q}}$. Further $\lambda \leq[\mu, f]$. To see that $\mu$ is an $S-K$ metric. we first observe that each $M_{\rho_{\gamma}}$ is automatically upper semi-continuous. Suppose that $M_{p q}(0) \neq 0$. If we fix $r, 0<r<1$, and take $u$ as a continuous function on $\overline{\Delta(0 ; r)}$ which dominates $\log M_{p_{q}}$ on $C(0 ; r)$ and is $C^{\prime \prime}$ and satisfies $\Delta u=4 e^{2 u}$ in $\Delta(0 ; r)$, we see that

$$
u(z) \geq \log M\left(z ; \varphi_{q}, \Omega\right), \quad|z|<r,
$$

and consequently

$$
u(z) \geq \log M_{\varphi q}(z), \quad|z| \leq r .
$$

From

$$
m(r ; u)-u(0) \geq e^{2 u(0)} r^{2}
$$

we conclude by the arbitrariness of $u$ that

$$
m\left(r ; \log M_{\varphi_{q}}\right)-\log M_{\varphi_{q}}(0) \geq\left[M_{\varphi_{q}}(0)\right]^{2} r^{2} .
$$

Consequently $\mu$ is an S-K metric. Part (a) of the theorem is established.

To establish (b) we note that the argument just employed shows the existence of an $S$ - $K$ metric on $G$.

Part (a) of Theorem 25.1 admits the following extension: Let $E$ denote a discrete subset of $G$ and let $\lambda$ denote a $C^{\prime \prime}$ conformal metric of constant curvature -4 on $G-E$ and suppose that $\lambda$ is the maximal among conformal metrics of this type with index $\nu(q ; \lambda), q \in E$. Let $f_{1}$ denote the restriction of $f$ to $f^{-1}(G-E)$. Then $\left[\lambda, f_{1}\right]$ is the maximal $C^{\prime \prime}$ metric of constant curvature 
-4 on $f^{-1}(G-E)-\left\{n\left(p ; f_{1}\right)>1\right\}$ whose index at a point $p \in f^{-1}(E)$ is $n(p ; f) \nu[f(p) ; \lambda]-n(p ; f)+1$ and at a point $p, n\left(p ; f_{1}\right)>1$, is $1-n(p ; f)$.

Part (b) yields information on the distribution of the points at which a conformal map $f$ is ramified in the case that $f$ is a map locally of island type onto a Riemann surface that does not possess a hyperbolic metric. For the special case where the domain of $f$ is $\Delta^{\prime} 0 ; 1$ ), it would be of interest to study the growth of the Nevanlinna characteristic function of $f$, as well as of $f$ belonging to more special classes.

[The following remark is worth making. Let $G$ be a Riemann surface with hyperbolic metric $r$ and let $f$ be a conformal map of a Riemann surface $F$ into $G$. Then a necessary and sufficient condition that $[r, f]$ be the hyperbolic metric of $F$ is that $f$ be a covering of $G$ (i.e. locally simple and locally of island type). The sufficiency follows from Theorem 25.1 (a). It can be established as well with the aid of conformal universal coverings. The necessity can be established with the aid of conformal universal coverings. This remark must be well-known.]

26. We seek to bracket the class of conformal maps that enjoy the property stated in Theorem 25.1 (a). Much remains to be done in this direction. In the present section we give a bracketing from above.

Let $f$ denote an interior transformation of a surface $F$ into a surface $G$. We say that $q \in G$ is a point locally omitted by $f$ provided that either $q \in G-f(F)$ or else $q \in f(F)$ and there exists a region $\Omega, q \in \Omega$, such that for some component $\omega$ of $f^{-1}(\Omega)$, the restriction of $f$ to $\omega$ omits $q$.

We show

THEOREM 26.1: Let $f$ denote a conformal map of a Riemann surface $F$ into a Riemann surface $G$ that possesses a hyperbolic metric $r$. Suppose that $f$ has the property stated in Theorem 25.1 (a). Then $f$ has no locally omitted point.

Proof: Suppose that there is a locally omitted point. At all events, $f(F)$ $=G$. Otherwise the hyperbolic metric $\gamma^{\prime}$ of $f(F)$ would exceed $\gamma^{f(F)}$ and $\left[\gamma^{\prime}, f\right]$ $=[\gamma, f]$ so that $\gamma^{\prime}=\gamma^{f(F)}$. There exists a locally omitted value $q$, and a uniformizer $\varphi, \varphi(0)=q$, such that the domain of $\varphi$ is $\Delta(0 ; 2)$, that $f$ is not ramified over $\varphi[C(0 ; 1)]$, and that $f$ omits $q$ on $\omega$ for some component $\omega$ of $f^{-1}\{\varphi[\Delta(0 ; 1)]\}$. Let $\lambda$ denote the continuous conformal metric on $\overline{\varphi[\Delta(0 ; 1)]}$ 
- $\{q\}$ satisfying: (a) its restriction, $\lambda^{\prime}$, to $\varphi[\Delta(0 ; 1)]-\{q\}$ is $C^{\prime \prime}$ of constant curvature -4 and has index 1 at $q$, (b) its restriction to $\varphi[C(0 ; 1)]$ is equal to the restriction of $\gamma$ to $\varphi[C(0 ; 1)]$. Let $\mu$ denote the conformal metric on $F$ satisfying

$$
\mu^{\omega}=\left[\lambda^{\prime}, f_{\omega}\right]
$$

and

$$
\mu^{F-\omega}=[\gamma, f]^{F-\omega} .
$$

It is easily verified that $\mu$ is an $S-K$ metric. Further $\mu$ exceeds $[\gamma, f]$ at the points of $\omega$ where $f$ is not ramified and in addition has the same index as $[\gamma f$,$] at each point of the set \{n(p ; f)>1\}$. It follows that the upper envelope $\bar{\mu}$ of the Perron family on $\{n(p ; f)=1\}$ generated by $\mu$ restricted to $\{n(p ; f)$ $=1$ \} exceeds the restriction of $[\gamma, f]$ to $\{n(p ; f)=1\}$. Further the index of $\bar{\mu}$ at each point of $\{n(p ; f)>1\}$ is the same as that of $[r, f]$. The contradiction is manifest.

27. On a remark of Carathéodory. In his Conformal Representation [3, first ed. p. 29] Carathéodory considers an analytic function $\varphi_{n}$ of modulus less than one on $\Delta(0 ; 1)$, which is of constant valence $n$ on $\Delta(0 ; 1)$, which has a ramification of order $n-1$ over a given point $w_{0} \in \Delta(0 ; 1), w_{0} \neq 0$, and which is subject to the normalization $\varphi_{n}(0)=0, \varphi_{n}^{\prime}(0)>0$. There is precisely one such function. In $\$ 59$ [loc. cit.] Carathéodory shows by direct computation that $\varphi_{n}^{\prime}(0)>\varphi_{n+1}^{\prime}(0)$ for all $n$ and observes that the stated inequality "may perhaps rest upon some deeper, as yet unremarked, property of the transformations". In the present section we shall consider a problem that embraces the situation considered by Carathéodory and we shall see how Carathéodory's insight may be justified. What is lacking, of course, in Carathéodory's example is the possibility of factoring $\varphi_{n+1}$ in the sense of composition in the form $\psi \circ \varphi_{n}$ where $\psi$ is an analytic function of modulus less than one on $\Delta(0 ; 1)$ and $\psi(0)=0$.

We consider Riemann surfaces $F, G, H$. Let $G$ possess a hyperbolic metric. We also consider a conformal map $f$ of $F$ into $H$ and a conformal map $g$ of $G$ into $H$. The following conditions are to be fulfilled:

(a) $g$ is to be locally of island type.

(b) The set $E$ of points over which $g$ is ramified is discrete and

$$
N(q)=\sup _{g(p)=q} n(p ; g)<+\infty, \quad q \in E .
$$


[The possibility that $E=\varnothing$ is allowed.]

(c) If $f(p) \in E, n(p ; f) \geq N[f(p)]$.

Under these conditions we show

THEOREM 27.1: F admits a hyperbolic metric. Further for each pair $(a, b)$ $\in F \times G$ satisfying $f(a)=g(b)$, there exists a quadruple $(K, k, \varphi, \psi)$ where $K$ is a Riemann surface, $k \in K, \varphi$ is a conformal map of $K$ into $F$, and $\psi$ is a conformal map of $K$ into $G$ for which the following conditions are fulfilled:

(1) $\varphi(k)=a, \phi(k)=b$.

(2) $f \circ \varphi=g \circ \psi$.

(3) $\varphi$ is a conformal map of $K$ onto $F$ which is locally of island type and which is such that if $n(p ; \varphi)>1$, then $f \circ \varphi(p) \in E$ and $n(p ; \varphi) \leq N[f \circ \varphi(p)]$.

(4) The inequality

$$
\left[\gamma_{1}, \varphi\right] \geq\left[\gamma_{2}, \psi\right]
$$

holds. $\gamma_{1}$ and $\gamma_{2}$ being respectively the hyperbolic metrics for $F$ and $G$.

Furthermore, if $F=G=\Delta(0 ; 1)$, then equality in (27.1) implies that $f=g \circ T$ where $T$ is a conformal automorphism of $\Delta(0 ; 1)$.

Before we turn to the proof, let us see how the observation of Carathéodory may be explained in the light of the present theorem. To do this, we identify $\varphi_{n+1}$ with $f$ and $\varphi_{n}$ with $g$. From (2), (4) and the last sentence of the theorem, we conclude that $\varphi_{n+1}^{\prime}(0)<\varphi_{n}^{\prime}(0)$. To be sure, this way of arriving at the inequality is far from elegant, but it does show that the phenomenon in question is a special instance of a fairly general situation.

Proof: It will be convenient to have available some well-known facts concerning analytische Gebilde for maps into Riemann surfaces. They are not novel but they do not appear to have been explicitly formulated. We start with given Riemann surfaces. For the purposes of the immediate consideration we shall call them $F$ and $G$ without implying that any restrictions are imposed. We consider ordered pairs $(\alpha, \beta)$, where $\alpha$ and $\beta$ have a common domain, a plane region containing 0 , and $\alpha$ and $\beta$ are conformal maps of this region into $F$ and $G$ respectively, such that

$$
z \rightarrow(\alpha(z), \beta(z))
$$


is univalent. An equivalence relation is defined in the class of such pairs as follows. Given two such pairs $\left(\alpha_{k}, \beta_{k}\right), k=1,2$, we say that $\left(\alpha_{2}, \beta_{2}\right)$ is "equivalent" to $\left(\alpha_{1}, \beta_{1}\right)$ provided that there exists $\varphi$ analytic at $0, \varphi(0)=0$, such that $\alpha_{1}[\varphi(z)]=\alpha_{2}(z), \beta_{1}[\varphi(z)]=\beta_{2}(z)$ for $z$ sufficiently small. We let $e(\alpha, \beta)$ denote the equivalence class containing $(\alpha, \beta)$ and we let $E$ denote the set whose elements are the $e(\alpha, \beta)$. Exactly as in the standard theory of analytische Gebilde we introduce as a topology in $E$ the weakest topology rendering each of the maps

$$
\theta_{\alpha \beta}: t \rightarrow e\left(\alpha^{t}, \beta^{t}\right), \quad t \in \text { domain of } \alpha \text { and } \beta,
$$

open, $\alpha^{t}$ and $\beta^{t}$ being defined by $\alpha^{t}(z)=\alpha(t+z), \beta^{t}(z)=\beta(t+z), z \in$ translate by $-t$ of the domain of $\alpha$ and $\beta$. Each $\theta_{\alpha \beta}$ is continuous. A component $\mathfrak{A}$ of $E$ is endowed with a conformal structure by the family of the $\theta_{\alpha \beta}$ having image in $\mathfrak{A}$. As in the classical case, we introduce the maps

$$
\left\{\begin{array}{l}
c: e(\alpha, \beta) \rightarrow \alpha(0), \\
v: e(\alpha, \beta) \rightarrow \beta(0),
\end{array}\right.
$$

and see that they are conformal maps of $\mathfrak{A}$ into $F$ and $G$ respectively. We term the components $\mathfrak{A}$ Gebilde, $c$ the center map, and $v$ the value map. [The terms center and value are adopted from the "Analytic Functions" of Saks and Zygmund.]

We now return to the situation of Theorem 27.1. Suppose that $f \circ c(p)$ $=g \circ v(p)$ for $p$ in the neighborhood of a point of $\mathfrak{A}$. Then $f \circ c=g \circ v$.

It is now easy to see that there exists $(\alpha, \beta)$ satisfying

$$
\alpha(0)=a, \beta(0)=b, f \circ \alpha=g \circ \beta,
$$

and further that the number of distinct equivalence classes of such pairs is precisely $n(b ; g) / m$ where $m$ is the smallest positive integer $l$ satisfying $n(b ; g) \mid \ln (a ; f)$. We fix such a class and term it $k$ and let $K$ denote the component of $E$ containing $k$ and let $\varphi=c$ and $\psi=v$. It is obvious that (1) and (2) of Theorem 27.1 are fulfilled.

To continue, we show that the covering properties of $g$ induce corresponding properties for $\varphi$. Given $p \in F$, there exists a disk $\Delta$ containing $p$ such that $f_{\Delta}$, the restriction of $f$ to $\Delta$, is a map of constant valence $n(p ; f)$ of $\Delta$ onto $f(\Delta)$ which is ramified at most at $p$, and on each component $\Omega$ of $g^{-1}[f(\Delta)]$, 
$g_{\Omega}$ is a map of constant valence with image $f(\Delta)$ which carries precisely one point $q \in \Omega$ into $f(p)$ and is ramified at no other point of $\Omega$. We now see that if $\Omega$ is given, there are precisely $n(q ; g) / m$ distinct equivalence classes of $(\alpha, \beta)$. satisfying: (i) $f \circ \alpha=g \circ \beta$, (ii) $\alpha(0)=p, \beta(0)=g$. Here $m$ is the smallest positive integer $l$ satisfying $n(q ; g) \mid l n(p ; f)$. There exist such $(\alpha, \beta)$ satisfying: (iii) the image of $\alpha$ is $\Delta$, the image of $\beta$ is $\Omega$, (iv) the domain of $\alpha$ and $\beta$ is $\Delta(0 ; 1)$ and $\alpha$ and $\beta$ are of constant valence on their images.

We shall conclude that $\varphi(K)=F$. At all events, $\varphi(K)$ is open. Suppose that $p \in \overline{\varphi(\bar{K})}$. There exists $r \in \Delta \cap \varphi(K), r \neq p ; \Delta$ is here construed in the sense of the previous paragraph. Let $r=\varphi(s)$. From $f[\varphi(s)]=g[\phi(s)]$ we see that there exists $\Omega, \psi(s) \in \Omega$. One of the $(\alpha, \beta)$ satisfying all the conditions (i)-(iv) has the property that $s=e\left(\alpha^{t}, \beta^{t}\right)$ for some $t,|t|<1$. With $(\alpha, \beta)$ so chosen, the image of $\theta_{\alpha \beta}$ is contained in $K$. Consequently $p \in \Delta \subset \varphi(K)$. Hence $\varphi(K)$ is closed. We conclude that $\varphi(K)=F$.

We now verify that $\varphi$ is locally of island type. Given $p \in F, \varphi(s)=p$, let $A$ be associated with $p$ as above. There exists $(\alpha, \beta) \in s$ satisfying (i), (iii), (iv). Consequently, $\left\{e\left(\alpha^{t}, \beta^{t}\right)|| t \mid<1\right\}$ is a component of $\varphi^{-1}(\Delta)$. Further, each component of $\varphi^{-1}(\Delta)$ is a set of this kind for some $s$. Hence, if $\Delta_{t}$ is a relatively compact disk. $p \in \Delta_{1} \subset \bar{\Delta}_{1} \subset \Delta$, then each component of $\varphi^{-1}\left(\Delta_{1}\right)$ is relatively compact.

Suppose that $n(s ; \varphi)>1$. From the definition of $K$, we see that with $p=\varphi(s), q=\phi(s)$, we have $n(q ; g)>1$. Hence $f(p) \in E$. From $n(s ; \varphi)$ $\leq n(q ; g), n(s ; \varphi) \leq N[f(p)]$. We conclude $(3)$.

The proof of (4) will be based on property (c) [stated before the theorem] which implies that $n(r ; \varphi) \leq n(s ; \varphi), s \in K$. From Theorem 25.1 (b) we conclude on considering $\left[\gamma_{2}, \psi\right]$ and $\varphi$ that $F$ possesses a hyperbolic metric $\gamma_{1}$ and we conclude (27.1) from Theorem 25.1 (a).

The final assertion of the theorem is immediate.

An application. Suppose that $H$ is the extended plane or the finite plane and that $F=G=\Delta(0 ; 1)$. We shall obtain from (27.1) inequalities connecting derivatives (resp. Laurent coefficients) of $f$ and $g$ at points $a$ and $b$ respectively where $f(a)=g(b)$. Let us first suppose that $f(a) \neq \infty$ and let $l=n(b ; g)$. Let $A$ denote the Taylor coefficient of $f$ of order $l$ at $a$ and let $B$ denote the Taylor coefficient of $g$ of order $l$ at $b$. Then from the local behavior of the solutions 
of $f(z)=g(w)$ and $(27.1)$ we obtain

$$
|A|^{1 / l}\left(1-|a|^{2}\right) \leq|B|^{1 / l}\left(1-|b|^{2}\right) .
$$

In particular, if $a=b=0$ and $n(0 ; g)=1$, we obtain

$$
\left|f^{\prime}(0)\right| \leq\left|g^{\prime}(0)\right| \text {. }
$$

It is to be observed that if (27.5) reduces to an equality, then Theorem 27.1 and the observation at the end of $\S 18$, Case 1 , assure us that $f=g \circ T$, where $T$ is a conformal automorphism of $\Delta(0 ; 1)$.

If $f(a)=\infty$, it suffices to consider the reciprocals of $f$ and $g$ respectively to deduce that either $n(a ; f)>l$ or $n(a ; f)=l$ and

$$
|A|^{-1 / l}\left(1-|a|^{2}\right) \leq|B|^{-1 / l}\left(1-|b|^{2}\right)
$$

where now $A$ is the Laurent coefficient of $f$ at $a$ of order $-l$ and $B$ is the Laurent coefficient of $g$ at $b$ of order $-l$.

The case of equality in (27.6) also yields the result that $f=g \circ T, T$ a conformal automorphism of $\Delta(0 ; 1)$.

28. An extension of Nevanlinna's criterion for hyperbolic type. Let $a_{1}$, $\ldots, a_{n}$ denote $n$ distinct points of the extended plane and let $\nu_{1}, \ldots, \nu_{n}$ denote positive integers $\geq 2$ or $+\infty$ satisfying

$$
\sum\left(1-\nu_{k}^{-1}\right)>2 \text {. }
$$

A celebrated theorem of Nevanlinna $[10 ;$ p. 282$]$ implies as an immediate corollary the result:

If a non-constant meromorphic function $f$ on $\Delta(0 ; R), R \leq+\infty$, satisfies

$$
n(z ; f) \geq \nu_{k},
$$

when $f(z)=a_{k}, k=1, \ldots, n$, then $R<+\infty$.

It is of interest to note that one may obtain this result as a corollary of a monotoneity property of induced metrics. Let $\lambda$ denote the Schwarz-Picard metric for the extended plane whose index $\alpha_{k}$ at $a_{k}$ is $1-\nu_{k}^{-1}$. Let $f$ denote a non-constant meromorphic function on a non-compact Riemann surface. Let $E=f^{-1}\left(\left\{a_{1}, \ldots, a_{n}\right\}\right)$. Let $\Omega$ denote a relatively compact Jordan region in $F$. Let 


$$
S_{\mathcal{Q}}(p)=\sum_{k=1}^{n}\left\{\sum_{f(q)=a_{k}}\left[1-n(q ; f) \nu_{k}^{-1}\right]^{+} g_{\Omega}(p, q)\right\}
$$

where $g$ is Green's function for $\Omega, p \in \Omega-E$. Let $\tilde{f}$ denote the restriction of $f$ to $F-E$. Then

$$
\exp \circ\left(-S_{\Omega}\right)[\lambda, \tilde{f}]_{\Omega-z}
$$

is an $S \cdot K$ metric and is the restriction to $\Omega-E$ of an $S-K$ metric $\mu_{\Omega}$ on $\Omega$. If $\Omega_{1}$ and $\Omega_{2}$ are admitted $\Omega$ satisfying $\Omega_{1} \subset \Omega_{2}$, then

$$
\mu_{\Omega_{1}} \geq\left(\mu_{\Omega_{2}}\right)^{\Omega_{1}}
$$

We conclude

THEOREM 28.1: If there exists a monotone increasing sequence of $\Omega$, say $\left(\Omega_{n}\right)_{1}^{\infty}$ exhausting $F$ such that for some point $a \in \Omega_{1}$ we have $\sup S_{\Omega_{n}}(a)<+\infty$, then $F$ has a hyperbolic metric. If the given condition is fulfilled non-trivially, then $F$ possesses Green's function.

We note that the hypotheses of Theorem 28.1 are automatically fulfilled in the situation of Nevanlinna's type criterion.

Similar results hold also for conformal maps with image in a torus.

Chapter IV. Applications. Meromorphic Functions on $\Delta(0 ; 1)$. Fuchsoid Groups

29. Our starting point will be a theorem connecting maximal $C^{\prime \prime}$ metrics of constant curvature -4 with assigned negative integral indices and bounded analytic functions on $\Delta(0 ; 1)$.

THEOREM 29.1: Let $E$ denote a non-empty discrete set in $\Delta(0 ; 1)$ and let $\lambda$ denote the maximal $C^{\prime \prime}$ conformal metric of constant curvature -4 on $\Delta(0 ; 1)-E$ with assigned negative integral indices at points of $E$, the class of such conformal metrics being assumed not empty. Then there exists an analytic function $f$ on $\Delta(0 ; 1)$ of modulus less than one such that

$$
\frac{\left|f^{\prime}(z)\right|}{1-|f(z)|^{2}}=\lambda(z), \quad z \in \Delta(0 ; 1)-E
$$

If $g$ also satisfies the same condition as $f$, then $g=T \circ f$ where $T$ is a conformal automorphism of $A(0 ; 1)$. 
Proof: We start with the case where $E$ is finite and the index at each point of $E$ is equal to -1 . In order to treat this case we proceed as follows. Let $A$-denote the set of ordered $n$-tuples $\left(a_{1}, \ldots, a_{n}\right)$ satisfying: $\left|a_{k}\right|<1$, $k=1, \ldots, n(\geq 1)$, and $a_{j} \neq a_{k}$ for $j \neq k$. It is easy to see that $A$ is an open connected subset of

$$
\underbrace{\Delta(0 ; 1) \times \cdots \times \Delta(0 ; 1)}_{n}
$$

Further let $B$ denote the subset of $A$ consisting of the $\left(a_{1}, \ldots, a_{n}\right)$ for which there exists a finite Blaschke product of degree $n+1$ of whose derivative the zeros in $\Delta(0 ; 1)$ consist precisely of $a_{1}, \ldots, a_{n}$. It is easy to see from examples that $B$ is not empty.

We next show that $B$ is open. Given $\left(a_{1}, \ldots, a_{n}\right) \in B$, let $f$ denote a finite Blaschke product of degree $n+1$ of whose derivative the zeros in $\Delta(0 ; 1)$ consist of $a_{1}, \ldots, a_{n}$. Let $w$ satisfy: $w \neq f\left(a_{k}\right), k=1, \ldots, n ;|w|<1$. Let $\alpha_{1}, \ldots, \alpha_{n+1}$ denote the distinct solutions of $f(z)=w$. The function $f$ admits a representation of the form

$$
f(z)=M\left(\eta \prod_{1}^{n+1} \cdot \frac{z-\alpha_{k}}{1-\bar{\alpha}_{k} z}\right),
$$

where $\eta$ is a complex number of modulus one and

$$
M(z)=\frac{z+w}{1+\bar{w} z}
$$

Let

$$
f_{\zeta}(z)=M\left[\eta\left(\prod_{1}^{n} \frac{z-\zeta_{k}}{1-\bar{\zeta}_{k} z}\right) \frac{z-\alpha_{n+1}}{1-\bar{\alpha}_{n+1} z}\right]
$$

where $\zeta=\left(\zeta_{1}, \ldots, \zeta_{n}\right)$. There exist disjoint neighborhoods of the $a_{k}$, say $N\left(a_{k}\right), k=1, \ldots, n$, and a neighborhood $N(\alpha)$ of $\alpha=\left(\alpha_{1}, \ldots, \alpha_{n}\right)$ of the form

$$
\Delta\left(\alpha_{1} ; r\right) \times \cdots \times \Delta\left(\alpha_{n} ; r\right)
$$

where the $\Delta\left(\alpha_{k} ; \gamma\right)$ are mutually disjoint, such that for each $\zeta \in N(\alpha)$ there are $n$ distinct zeros of $f_{\zeta}^{\prime}$ in $\Delta(0 ; 1)$, say $b_{k}(\zeta), k=1, \ldots, n$ where $b_{k}(\zeta) \in N\left(a_{k}\right)$. The mapping

$$
\zeta \rightarrow\left(b_{4}(\zeta), \ldots, b_{n}(\zeta)\right), \quad \zeta \in N(\alpha)
$$


is $(1,1)$ and continuous. The continuity is elementary. If $\zeta, \omega \in N(\alpha)$ go into the same point under the map (29.4), then by Theorem 25.1 (a), we have

$$
\underset{1-\left|f_{\zeta}\right|^{2}}{\left|f_{\zeta}^{\prime}\right|}=\frac{\left|f_{w}^{\prime}\right|}{1-\left|f_{w 0}\right|^{2}}
$$

Consequently $f_{\zeta}=S \circ f_{\omega}$ where $S$ is a conformal automorphism of $\Delta(0 ; 1)$ with fixed point $w$. Hence $\left\{\zeta_{1}, \ldots, \zeta_{n}\right\}=\left\{\omega_{1}, \ldots, \omega_{n}\right\}$ and by the disjunction of the $\Delta\left(\alpha_{k} ; r\right)$ we have $\zeta=\omega$. Brouwer's theorem of Gebietstreue [8] now yields the conclusion that $\left(a_{1}, \ldots, a_{n}\right) \in$ int $B$. Hence $B$ is open.

To show that $B$ is closed in the sense of the relative topology of $A$ we proceed as follows. Given $a^{0}=\left(a_{1}^{0}, \ldots, a_{n}^{0}\right) \in \bar{B} \cap A$. There exists a sequence $\left(a^{m}\right)_{1}^{\infty}, a^{m} \in B$, with limit $a^{0}$. Let $g_{m}$ denote a finite Blaschke product of degree $n+1$ of whose derivative the zeros in $\Delta(0 ; 1)$ consist of the components of $a^{m}, g_{m}(0)=0$. There exists a convergent subsequence of $\left(g_{m}\right)$ with limit $g$. It is easy to see that $g$ is a finite Blaschke product of degree $n+1$ of whose derivative the zeros in $\Delta(0 ; 1)$ consist of the components of $a^{0}$. Consequently $a^{0} \in B . \quad B$ is closed in the sense of the relative topology of $A$. Hence $B=A$. The theorem is thereby cared for in the case where $E$ is finite and the index is -1 at each point of $E$.

To treat the case where $E$ is finite but the indices are unrestricted negative integers, it suffices to introduce a sequence $\left(g_{n}\right)$ of finite Blaschke products, each of degree $1-\sum \nu(q), \nu(q)$ being the value assigned for the index at $q \in E$, $g_{n}(0)=0$, such that the zeros of $g_{n}^{\prime}$ in $\Delta(0 ; 1)$ are simple and tend to the points of $E,-{ }_{\nu}(q)$ of them tending to $q$, and thereupon to observe that the limit of a convergent subsequence of $\left(g_{n}\right)$ serves.

To treat the case where $E$ is infinite, let $\left(E_{n}\right)_{1}^{\infty}$ denote a monotone increasing sequence of sets with union $E$. Let $g_{n}$ denote an analytic function of modulus less than one on $\Delta(0 ; 1), g_{n}(0)=0$, which has the required property relative to $E_{n}$ and the values assigned for the indices. Now

$$
\left|g_{n}^{\prime}\right|\left(1-\left|g_{n}\right|^{2}\right)^{-1}
$$

is monotone non-increasing and tends to $\lambda$. On the other hand, there exists a subsequence of $\left(g_{n}\right)$ which tends to an analytic function $g$ of modulus less than one on $\Delta(0 ; 1), g(0)=0$. It follows that 


$$
\frac{\left|g^{\prime}(z)\right|}{1-|g(z)|^{2}}=\lambda(z)
$$

The lasst sentence of the theorem does not call for special details.

30. The distribution of the ramification points of a Lindelöfian map. Thanks to Theorem 29.1 we see that there is a very close connection between the derivatives of bounded analytic functions on $\Delta(0 ; 1)$ and the conformal metrics of Theorem 29.1. We introduce a divisor $\partial$ on a Riemann surface $F$ as a real-valued function on $F$ such that $\{\partial(p) \neq 0\}$ is discrete. Given a conformal map $f$ with domain $F$, by the ramification divisor $\partial_{f}$ of $f$ is meant the function on $F$ defined by

$$
\partial_{f}(p)=n(p ; f)-1
$$

Given a $C^{\prime \prime}$ conformal metric $\lambda$ of constant curvature -4 on $F-E$, where $E$ is a discrete subset of $F$, by the divisor of $\lambda, \partial_{\lambda}$, is meant the function with domain $F$ defined by

$$
\partial_{\lambda}(p)=\left\{\begin{array}{l}
0, p \in F-E ; \\
\nu(p ; \lambda), p \in E .
\end{array}\right.
$$

It is now immediate that the following theorem holds.

Theorem 30.1: The set $\left\{\partial_{\lambda}\right\}, \lambda$ admitted by Theorem 29.1, is the same as the set $\left\{-\partial_{f}\right\}, f$ bounded analytic not constant on $\Delta(0 ; 1)$.

We may go further and study the ramification divisors of Lindelöfian maps [7] having domain $d(0 ; 1)$. Let us recall that a Lindelöfian map may be defined as a conformal map $f$ of a Riemann surface $F$ possessing Green's function $g$ into a Riemann surface $G$ which satisfies

$$
S_{q}(p) \equiv \sum_{f(r)=q} n(r ; f) g(p, r)<+\infty, \quad f(p) \neq q .
$$

In case $G$ is the extended plane and $F=\Delta(0 ; 1)$ the requirement that $f$ be Lindelöfian is equivalent to the condition that it be of bounded type in the sense of R. Nevanlinna. [Indeed, in the unrestricted case the requirement that a map be Lindelöfian is equivalent to its having bounded Nevanlinna characteristic function in the sense of an extension of Nevanlinna's theory to conformal maps of Riemann surfaces $[7,12]$.]

We show 
TheOREM 30.2: Given a Riemann surface $G$. The set D of ramification divisors of Lindelöfian maps of $\Delta(0 ; 1)$ into $G$ is the same as the set $\left\{\partial_{f}\right\}, f$ analytic, not constant, and of modulus $<1$ on $\Delta(0 ; 1)$.

Proof: Given a member of the second class $\partial_{f}$, it suffices to take a univalent conformal map $\varphi$ of $\Delta(0 ; 1)$ into $G$ to obtain in $\varphi \circ f$ a Lindelöfian map of $\Delta(0 ; 1)$ into $G$ satisfying $\partial_{p \circ f}=\partial_{f}$. Hence the latter set of divisors of the theorem is contained in the former.

Suppose now that $g$ is a Lindelöfian map of $\Delta(0 ; 1)$ into $G$. Let $a, b, c$ denote three distinct points of $G$ over which $g$ is not ramified. Let $r$ denote the hyperbolic metric for $G-\{a, b, c\}$. Let $g^{*}$ denote the restriction of $g$ to $g^{-1}(G-\{a, b, c\})$. Referring to $(30.3)$ with $F=\Delta(0 ; 1)$ and with our present $g$ in place of $f$, we introduce

$$
\exp \circ\left[-\left(S_{a}^{*}+S_{b}^{*}+S_{c}^{*}\right)\right]\left[\gamma, g^{*}\right]
$$

where $S_{q}^{*}$ is taken as the restriction to $g^{-1}(G-\{a, b, c\})$ of $S_{q}$. The metric (30.4) is an $S-K$ metric on $g^{-1}(G-\{a, b, c\})$. Let $\mu$ denote the restriction to $\left\{\partial_{g}(z)=0\right\}$ of the continuous conformal metric on $\Delta(0 ; 1)$ whose restriction to $g^{-1}(G-\{a, b, c\})$ is the conformal metric (30.4). Then the upper envelope $\lambda$ of the Perron family generated by $\mu$ satisfies $\partial_{\lambda}=-\partial_{g}$. Hence by Theorem $30.1, \mathfrak{D} \subset\left\{\partial_{f}\right\}$. Theorem 30.2 follows.

We remark that the latter part of the theorem could also have been demonstrated by observing that given a Lindelöfian map $f$ with domain $\Delta(0 ; 1)$ there exists a function of bounded type with domain $\Delta(0 ; 1)$ which has the same ramification divisor as $f$. However the basic argument is not altered thereby.

The following evident remark should be made. If $G$ admits a conformal universal covering with domain $\Delta(0 ; 1)$, then the set of ramification divisors of conformal maps of $\Delta(0 ; 1)$ into $G$ is the same as the set of ramification divisors of non-constant bounded analytic functions on $\Delta(0 ; 1)$.

Theorem 30.2 permits us to characterize the distribution of the $a$-points of the derivative of a function of bounded type. We recall that the sum of two meromorphic functions of bounded type is itself of bounded type if it is not constant. Given $a$ finite and $g$ meromorphic of bounded type on $\Delta(0 ; 1)$, we introduce

$$
h_{a}(z)=g(z)-a z
$$


and notice that, with $\delta_{a}(z)$ denoting the multiplicity of $g^{\prime}$ at $z$ if $g^{\prime}(z)=a$ and otherwise zero,

$$
\delta_{a} \leq \partial_{h_{b}}, \quad\left(g^{\prime}(z) \neq a\right) .
$$

We conclude

THEOREM 30.3: Let $g$ be a non-constant meromorphic function of bounded type on $\Delta(0 ; 1)$ and let $a$ be $a$ finite number. If $g^{\prime}(z) \neq a$, $\delta a$ is the ramification divisor of a non-constant bounded analytic function on $\Delta(0 ; 1)$.

The converse result is immediate: given $a$ finite and $\partial$ the ramification divisor of a non-constant bounded analytic function, there exists a meromorphic function $g$ of bounded type on $\Delta(0 ; 1)$ for which $\delta_{a}=\partial$. The characterization of the distribution of the poles of the derivative of a function of bounded type on $\Delta(0 ; 1)$ is so obvious (in terms of the Blaschke condition) that we omit its consideration.

31. Growth problems. It is easy to obtain upper estimates on the growth of the Anzahlfunktion for the zeros of the derivative of a non-constant bounded analytic function on $\Delta(0 ; 1)$, even by means of classical function-theoretic methods. It is of interest to see to what extent the growth estimates so obtained are sharp and here the results of the present paper will be seen to be useful.

Let $f$ denote a non-constant analytic function of modulus less than one on $\Delta(0 ; 1)$ and let

$$
n_{1}(r ; f)=\sum_{|z|<r} \partial_{f}(z), \quad 0 \leq r<1
$$

and

$$
N_{1}(r ; f)=\left\{\begin{array}{l}
\int_{0}^{r} t^{-1} n_{1}(t) d t \quad \text { if }{ }^{\circ} \partial_{f}(0)=0 ; \\
\int_{0}^{r} t^{-1}\left[n_{1}(t)-\partial_{f}(0)\right] d t+\partial_{f}(0) \log r \quad \text { if } \partial_{f}(0)>0 .
\end{array}\right.
$$

By the Schwarz-Pick lemma we obtain

$$
\log \left[\frac{\left|f^{\prime}(z)\right|}{1-|f(z)|^{2}}\left|+\sum n\left(t ; f^{\prime}\right) \log \right| \frac{r^{2}-\bar{t} z}{r(z-t)}\left|\leq \log \frac{1}{1-r^{2}}, \quad\right| z \mid<r,\right.
$$

where the sum is taken over $t$ satisfying: $|t|<r$ and $f^{\prime}(t)=0$. Hence

$$
N_{1}(r ; f) \leq \log \frac{1}{1-r}+A, \quad 0 \leq r<1,
$$


$A$ being constant. We conclude

$$
\limsup _{r \rightarrow 1} \frac{N_{1}(r ; f)}{-\log (1-r)} \leq 1 .
$$

We shall now see that for the class of admitted $f$ the upper bound 1 cannot be improved. To that end, we let $p$ denote a positive integer $\geq 2$ and we let $\Gamma$ denote the Fuchsian group that leaves invariant a conformal universal covering of a compact Riemann surface of genus $p$. Let $n_{\Gamma}(r)$ denote the number of points in $\Delta(0 ; r)$ equivalent to $a$ with respect to $\Gamma, 0<r<1$, ( $a$ not equivalent to 0$)$ and let

$$
N_{\Gamma}(r)=\int_{0}^{r} t^{-1} n_{\Gamma}(t) d t, \quad 0<r<1
$$

A well-known result [17] yields

$$
\lim _{r \rightarrow 1} \frac{N_{\Gamma}(r)}{-\log } \frac{1}{(1-r)}=\frac{1}{2 p-2} .
$$

We know from the Schwarz-Picard existence theorem that there exists a $C^{\prime \prime}$ metric of constant curvature -4 on $\Delta(0 ; 1)$ less the orbit of $\Gamma$ containing $a$ having index $3-2 p$ at each point of the orbit. By Theorem 29.1 we see that there exists an analytic function $f$ of modulus less than one on $\Delta(0 ; 1)$ satisfying

$$
\lim _{r \rightarrow 1} \frac{N_{1}(r ; f)}{-\log (1-r)}=\frac{2 p-3}{2 p-2} .
$$

Since we may take $p$ arbitrarily large, we see that (31.5) is the best possible inequality of its kind.

The example that we have produced gives another solution to the problem of Bloch which asks whether the derivative of a non-constant bounded analytic function on $\Delta(0 ; 1)$ is necessarily of bounded type. The problem was settled negatively by Frostman [6] who exhibited a Blaschke product the derivative of which fails to have the Fatou property. The work of the present section supplements the solution of Frostman in that it yields quantitative information on $N_{1}(r ; f)$ for non-constant bounded analytic $f$ on $\Delta(0 ; 1)$.

I have not determined whether there exists such an $f$ for which

$$
\lim _{r \rightarrow 1} \frac{N_{1}(r ; f)}{-\log \cdot(1-r)}=1
$$


It would also be of interest to study the dependence of $N_{1}(r ; f-a z)$ on $a$.

One must not expect that an arbitrary non-negative integer-valued divisor o on $\Delta(0 ; 1)$ is necessarily the ramification divisor of a non-constant bounded analytic function $f$ on $\Delta(0 ; 1)$ even if with $n_{\partial}(r)=\sum_{|z|<r} \partial(z)$ and $N_{\partial}(r)$ $=\int_{r_{0}}^{r} t^{-1} n_{\partial}(t) d t$ we have

$$
\limsup _{r \rightarrow 1} \frac{N_{\partial}(r)}{-\log (1-r)} \leq 1
$$

In fact, suppose that $g$ is an analytic function on $\Delta(0 ; 1)$ which is locally of island type with respect to the finite plane and is ramified precisely at the antecedents of $m+i n, m$ and $n$ being integers, and the multiplicity of $g$ at each such point being 2. By Bloch's theorem we obtain the inequality

$$
\left|g^{\prime}(z)\right| \leq \frac{B}{1-|z|^{2}}, \quad|z|<1,
$$

where $B$ is a positive number. We take $\partial=\partial_{g}$. From (31.10) we see that (31.5) holds, $g$ replacing $f$. However there does not exist a bounded analytic function $b$ on $J(0 ; 1)$ satisfying $\partial_{b}=\partial$. Otherwise from Theorem 25.1 (b) we should infer that the finite plane possesses a hyperbolic metric.

32. The property of $S \cdot K$ metrics given in $\S \mathbf{1 0}$ (e) permits us to obtain information concerning analytic functions on $\Delta(0 ; 1)$ which do not grow too rapidly. Let $A$ and $a$ be positive numbers. Let $f$ be analytic on $\Delta(0 ; 1)$ and not constant, and let $f$ satisfy

$$
|f(z)| \leq A(1-|z|)^{-a}, \quad|z|<1 .
$$

It follows from $\$ 10$ (e) that for each $\eta, 0<\eta<1$, there exists a non-trivial $S-K$ metric $\lambda_{\eta}$ on $\Delta(0 ; 1)$ which has a zero of $\operatorname{order} a^{-1} \eta n(z ; f)$ at a zero $z$ of $f$.

Now suppose that $\mu$ is an $S-K$ metric on $\{\operatorname{Re} z>0\}$. We follow the convention put down earlier and use $\mu$ for the scale associated with the identity map on $\{\operatorname{Re} z>0\}$ as well. Now $\log \mu$ is subharmonic and

$$
\mu(z) \leq(2 \operatorname{Re} z)^{-1}, \quad \operatorname{Re} z>0,
$$

the right-hand side of the inequality being the scale of the hyperbolic metric corresponding to the identity map on $\{\operatorname{Re} z>0\}$. If, in particular, $\mu=\left[\lambda_{\eta}, T\right]$ where $T$ is a univalent conformal map of $\{\operatorname{Re} z>0\}$ onto $\Delta(0 ; 1)$, then the 
subharmonicity of $\log \mu$ and (32.2) imply

$$
\sum n[T(w) ; f] \log \left|\frac{z-2 u_{0}+\bar{w}}{z-w}\right| \neq+\infty, \quad \operatorname{Re} z>u_{0}>0,
$$

the summation being taken over $w$ satisfying $f[T(w)]=0, \operatorname{Re} w>u_{0}$. On returning to $A(0 ; 1)$, we see that the zeros of the restriction of $f$ to a disk whose frontier is an oricycle tangent to the unit circumference satisfy the Blaschke condition. What has been said for $f$ holds as well for $f-c$ where $c$ is an arbitrary complex constant. We are led at once to

THEOREM 32.1: The restriction of $f$ to $a$ disk whose frontier is an oricycle tangent to the unit circumference is a function of bounded type.

We need hardly remark that the derivative of a bounded analytic function on $\Delta(0 ; 1)$ satisfies $(32.1)$ with $a=1$.

Theorem 32.1 has the following consequence:

Let $r \rightarrow N(r)$ denote a non-decreasing function on $\{0 \leq r<1\}$ taking nonnegative integral values, $N(0)=0$, and $N$ being continuous on the left. If for every divisor $\partial$ taking non-negative integral values on $\Delta(0 ; 1)$ and satisfying

$$
\sum_{|z|<r} \partial(z)=N(r)
$$

there exists $f$ satisfying (32.1) for some $A$ and $a$ for which $n(z ; f)=\partial(z)$ when $\partial(z)>0$ and $f(z) \neq 0$ when $\partial(z)=0$, then

$$
\int_{r_{0}}^{1} t^{-1} N(t) d t<+\infty, \quad 0<r_{0}<1
$$

33. The class $C$ of the ramification divisors of functions $g$ analytic on $\Delta(0 ; 1), g^{\prime}$ satisfying $(32.1)$ with $a=1$, may be bracketed as follows. Let $A$ denote the set of the ramification divisors of the non-constant bounded analytic functions on $\Delta(0 ; 1)$. Let $0<\eta<1$ and let $B_{\eta}$ denote the class of divisors $\partial$ on $\Delta(0 ; 1)$ with non-negative integral values for which there exists a $C^{\prime \prime}$ conformal metric of constant curvature -4 on $\Delta(0 ; 1)-\{\partial(z)>0\}$ with index $-\eta \partial(z)$ for $z$ satisfying $\partial(z)>0$. Then

$$
A \subsetneq C \subset \bigcap_{0<\eta<1} B_{\eta} .
$$

The strong inclusion on the left follows from $\S 31$. It would be of interest to determine whether the inclusion on the right side of (33.1) is strong. 
34. The argument of $\S \mathbf{3 2}$ may be made to yield a general oricycle property of arbitrary Fuchsian or Fuchsoid groups. We recall that if $\Gamma$ is an arbitrary Fuchsian or Fuchsoid group acting on $\Delta(0 ; 1)$, then the set of orbits $\Gamma(z)$ $=\{\tau(z) \mid \tau \in \Gamma\}$ may be so endowed with a conformal structure that $z \rightarrow I(z)$ is rendered a conformal map. Such a conformal structure is essentially unique. Thanks to the results of $\S \mathbf{1 4}$ and the argument of $\S \mathbf{3 2}$ we conclude

THEOREM 34.1: The restriction of $z \rightarrow I^{\prime}(z)$ to the interior of an oricycle tangent to $C(0 ; 1)$ is a Lindelöfian conformal map.

35. The following theorem relates the ramification divisor of a meromorphic function totally ramified over a finite number of points to ramification divisors for bounded analytic functions. The notation $[\alpha]$ will be employed to denote the integral part of a real number $\alpha$.

TheOREm 35.1: Let $a_{1}, \ldots, a_{n}$ denote $n$ distinct points of the extended plane. Let $\nu_{1}, \ldots, \nu_{n}$ be real numbers, $\nu_{k}<1, k=1, \ldots, n$, sațisfying

$$
\sum_{k=1}^{n} \nu_{k}>2
$$

Let $f$ denote a non-constant meromorphic function on $\Delta(0 ; 1)$ satisfying $n(z ; f)$ $\geq 2$ when $f(z)=a_{k}, k=1, \ldots, n$. Let $\partial$ denote the divisor defined by

$$
\partial(z)=\left\{\begin{array}{l}
n(z ; f)-1, \text { when } f(z) \neq a_{1}, \ldots, a_{n} \\
n(z ; f)\left(1-\nu_{k}\right)-1, \text { when } f(z)=a_{k} .
\end{array}\right.
$$

If $\partial \geq 0$, then $\partial$ is the ramification divisor of some non-constant bounded analytic function on $\Delta(0 ; 1)$.

The proof is very simple. Let $\lambda$ denote the Schwarz-Picard metric on the extended plane less $\left\{a_{1}, \ldots, a_{n}\right\}$ with index $\nu_{k}$ at $a_{k}$. The negative of the divisor of $[\lambda, \tilde{f}]$ where $\tilde{f}$ is the restriction of $f$ to $\{n(z ; f)=1\}$ dominates $\partial$. It follows that there exists a $C^{\prime \prime}$ conformal metric of constant curvature -4 on $\Delta(0 ; 1)-\{\partial(z)>0\}$ with index $-\partial(z)$ when $\partial(z)>0$. The theorem now follows from Theorem 29.1.

A similar argument establishes

Theorem 35.2: Let $f$ denote a conformal map of $\Delta(0 ; 1)$ into a torus $T$ which has the property that there exists $a \in T$ such that $n(z ; f)>1$ when $f(z)$ 
$=a$. Then if $0<\eta<1$, the divisor $\partial$ defined by

$$
\partial(z)=\left\{\begin{array}{l}
n(z ; f)-1, \text { when } f(z) \neq a ; \\
n(z ; f)(1-\eta)-1, \text { when } f(z)=a,
\end{array}\right.
$$

is the ramification divisor of a bounded analytic function on $\Delta(0 ; 1)$ provided that $\partial \geq 0$.

\section{Chapter V. The Bloch constant exceeds $\sqrt{3} / 4$.}

36. Before we turn to the proof of the theorem stated in the heading, it will be convenient to recall some basic facts concerning the Bloch constant as well as the argument of Ahlfors which led to the result: the Bloch constant is at least $\sqrt{3} / 4$. Let $f$ denote a non-constant analytic function on a given Riemann surface $F$. By the Bloch number $b(f)$ of $f$ we understand the supremum of the set of positive $r$ for which there exists a region $\Omega(\subset F)$ which $f$ maps univalently onto a disk of radius $r$. By the Bloch constant $B$ we understand the infimum of the set of $b(f)$ when all $f$ analytic on $\Delta(0 ; 1)$ and satisfying $f^{\prime}(0)=1$ are taken into account.

In showing that $B \geq \sqrt{3} / 4$ Ahlfors proceeded (essentially) in the following way. For each $f$ analytic on $\Delta(0 ; 1)$ and satisfying $f^{\prime}(0)=1$, a function $\rho_{f}$ with domain $\Delta(0 ; 1)$ is introduced. When $f^{\prime}(z)=0, \rho_{f}(z)=0$. When $f^{\prime}(z) \neq 0$, then $\rho_{f}(z)$ is the supremum of the set of positive numbers $r$ such that the restriction of $f$ to the component $\Omega$ of $f^{-1}[\Delta(f(z) ; r)]$ containing $z$ maps $\Omega$ univalently onto $\Delta(f(z) ; r)$. In the latter case, $\rho_{f}(z)$ is actually the maximum of such $r$ and there exists $c$ satisfying $|c-f(z)|=\rho_{f}(z)$ such that

$$
\rho_{f}(t) \leq|f(t)-c|, \quad t \in \Omega_{z} .
$$

Further $\rho_{f}$ is continuous on $\Delta(0 ; 1)$. An essential part of Ahlfors's argument is the fact that when $b(f)<+\infty$ and $\sqrt{3 b(f)}<A<+\infty$

$$
\frac{A\left|f^{\prime}\right|}{2 \rho_{f}^{1 / 2}\left(A^{2}-\rho_{f}\right)}
$$

is (in our present terminology) the scale for an $S-K$ metric on $\Delta(0 ; 1)$ relative to the identity, the definition at a point $z$ where $f^{\prime}(z)=0$ being taken as the limit at the point. The inequality (36.1) and the restriction on $A$ are the basis for the support property [cf. §6 A 2] at the points $z$ where $f^{\prime}(z) \neq 0$. The 
inequality $B \geq \sqrt{3} / 4$ follows on noting that the value of (36.2) at 0 is no greater than one. This, in brief, is Ahlfors's argument.

Suppose that $B=\sqrt{3} / 4$. It is well known that there exists a function $g$ analytic on $\Delta(0 ; 1), g(0)=0, g^{\prime}(0)=1$, for which $b(g)=B$. For $g$ we should conclude that

$$
\frac{A\left|g^{\prime}\right|}{2 \rho_{g}^{1 / 2}\left(A^{2}-\rho_{g}\right)}, \quad A=\frac{3^{3 / 4}}{2},
$$

is the scale with respect to the identity of an $S-K$ metric on $\Delta(0 ; 1)$. The con vention made in connection with (36.2) holds where $g^{\prime}(z)=0$. Further

$$
\rho_{g}^{1 / 2}\left(A^{2}-\rho_{g}\right) \leq B^{1 / 2}\left(A^{2}-B\right)=\frac{3^{3 / 4}}{4} .
$$

It follows that (36.3) attains the value 1 at 0 , hence (36.3) is equal to $\left(1-|z|^{2}\right)^{-1}$ throughout $\Delta(0 ; 1)$. Further $\rho_{g}(0)=B$. We take (36.1) into account for $g$ with $z=0$ noting that $|c|=B$. Let $\Omega^{\prime}=\{|g(z)-c|<B\} \cap \Omega, \Omega$ being taken in the present context. We note that

$$
\rho_{g}(z) \leq|g(z)-c|<B, \quad z \in \Omega^{\prime},
$$

and that

$$
\rho_{g}(z)=|g(z)-c|, \quad z \in \Omega^{\prime} \cap g^{-1}(\overline{0 c})
$$

Hence

$$
\frac{A\left|g^{\prime}\right|}{2|g-c|^{1 / 2}\left(A^{2}-|g-c|\right)} \leq \frac{A\left|g^{\prime}\right|}{2 \rho_{g}^{1 / 2}\left(A^{2}-\rho_{g}\right)}
$$

in $\Omega^{\prime}$ and equality holds along an arc. Since each side of (36.7) defines in $\Omega^{\prime}$ a $C^{\prime \prime}$ conformal metric of constant curvature -4 , the two sides of (36.7) agree on $\Omega^{\prime}$. That is, the left hand side of (36.7) agrees with $\left(1-|z|^{2}\right)^{-1}$ on a non. empty open subset of $\Delta(0 ; 1)$.

We are led to the conclusion that

$$
g=c+A^{2} L^{2}
$$

where $L$ is a conformal automorphism of $\Delta(0 ; 1)$. But (36.8) implies that $b(g)=A^{2} / 2$, that is,

$$
\frac{3^{1 / 2}}{4}=\frac{3^{3 / 2}}{8}
$$


Since this is false, we conclude that $B>\sqrt{3} / 4$. Another reason why (36.8) cannot hold is furnished by the theorem of R. M. Robinson [14] which asserts that a Bloch extremal function has a natural boundary.

\section{BIBLIOGRAPHY}

[1] Ahlfors, L. V. An extension of Schwarz's Lemma. Trans. A.M.S. 43 (1938), 359-364.

[2] Bieberbach, L. Göttinger Nachrichten (1912), 599-602.

[ 3 ] Carathéodory, C. Conformal Representation. 1st ed. Cambridge, 1932.

[ 4 ] Courant, R. and Hilbert, D. Methoden der Mathematischen Physik. Vol. 2, Berlin, 1937.

[5] Deny, J. Sur les infinis d'un potentiel. C. R. Acad. Sci. Paris 224 (1947), 524-525.

[6] Frostman, O. Sur les produits de Blaschke. K. Fys. Sall. i Lund För. 12 (1943). Nr. $15,1-14$.

[ 7 ] Heins, M. Lindelöfian maps. Ann. Math. 62 (1955), 418-446.

[8] Hurewicz, W. and Wallman, H. Dimension Theory. Princeton, 1948.

[ 9 ] Nehari, Z. A generalization of Schwarz' Lemma. Duke M.J. 14 (1947), 1035-1049.

[10] Nevanlinna, R. Eindeutige analytische Funktionen. 2 d ed. Berlin, 1953.

[11] Nevanlinna, R. Le Theoreme de Picard-Borel et la Theorie des Fonctions Meromorphes. Paris, 1929.

[12] Parreau, M. Fonction caracteristique dune application conforme. Ann. Fac. Sci. Toulouse 19 (1956), 175̄-189.

[13] Picard, E. Traité d Analyse. vol. 3. Paris, 1928.

[14] Robinson, R. M. Bloch functions. Duke M. J. 2 (1936), 453-458.

[15] Robinson, R. M. A generalization of Picard's and related theorems. Duke M. J. 5 (1939), 118-131.

[16] Saks, S. and Zygmund, A. Analytic Functions Warsaw, Wroclaw, 1952.

[17] Tsuji, M. Potential Theory in Modern Function Theory. Tokyo, 1959.

[18] Virtanen, K. I. Eine Bemerkung über die Anwendung hyperbolischer Massbestimmungen in der Wertverteilungslehre der meromorphen Funktionen. Math. Scand. 1 (1953), 153-158.

University of Illinois 